1990

\title{
Aqueous Solubility of Liquid Hydrocarbon Mixtures Containing Dissolved Solid Components
}

George G. Vadas

College of William and Mary - Virginia Institute of Marine Science

Follow this and additional works at: https://scholarworks.wm.edu/etd

Part of the Physical Chemistry Commons

\section{Recommended Citation}

Vadas, George G., "Aqueous Solubility of Liquid Hydrocarbon Mixtures Containing Dissolved Solid Components" (1990). Dissertations, Theses, and Masters Projects. Paper 1539617610.

https://dx.doi.org/doi:10.25773/v5-avfm-2e15

This Thesis is brought to you for free and open access by the Theses, Dissertations, \& Master Projects at W\&M ScholarWorks. It has been accepted for inclusion in Dissertations, Theses, and Masters Projects by an authorized administrator of W\&M ScholarWorks. For more information, please contact scholarworks@wm.edu. 


\title{
AQUEOUS SOLUBILITY OF LIQUID HYDROCARBON MIXTURES CONTAINING DISSOLVED SOLID COMPONENTS
}

\begin{abstract}
A Thesis
Presented to

The Faculty of the School of Marine Science

The College of William and Mary in Virginia
\end{abstract}

In Partial Fulfillment

of the Requirements for the Degree of

Master of Arts

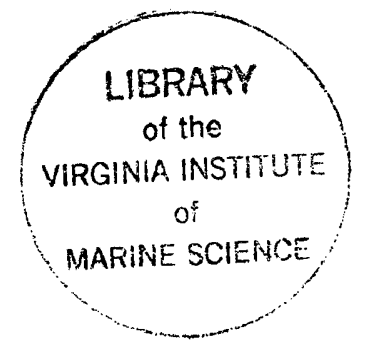

by

George G. Vadas 


\section{APPROVAL SHEET}

This thesis is submitted in partial fulfillment of the requirements for the degree of

\section{Master of Arts}

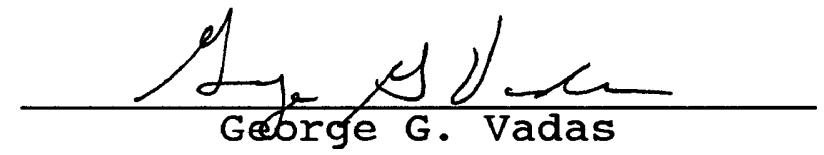

Approved, December 1990
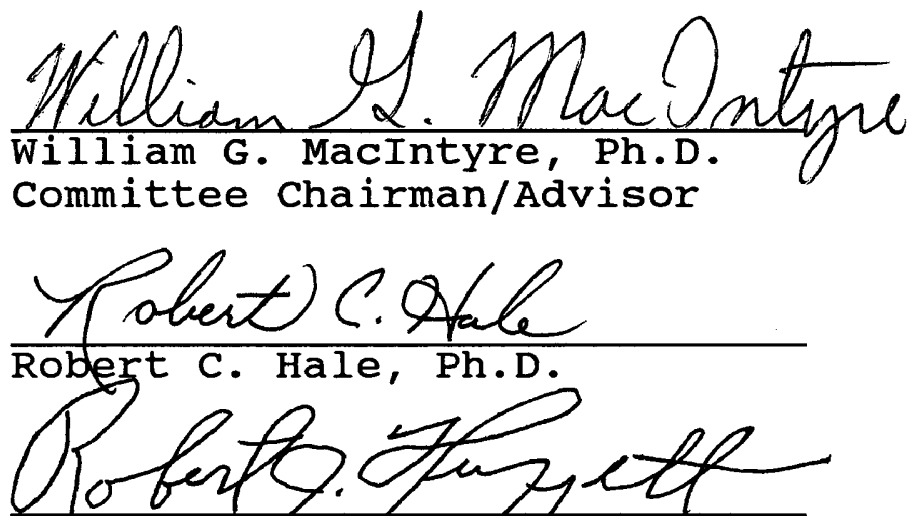

Robert s. Huggett/ph.D.

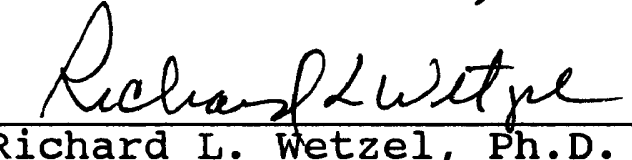

Richard L. Wetzel, Ph.D.

$\frac{\text { Mald W. Phm }}{\text { Gerald H. Bohnson, Ph.D. }}$

College of William and Mary

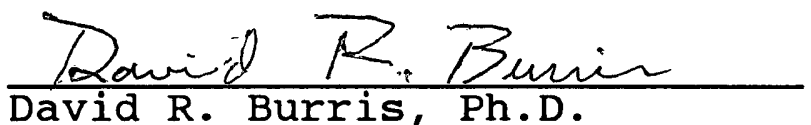

David R. Burris, Ph.D.

Engineering and Services Center Laboratory U.S. Air Force 


\section{Dedication}

This work is dedicated to my family and friends. Thank you for the understanding, kindness, and support you have given me over the years, and to little scro. 


\section{TABLE OF CONTENTS}

Page

ACKNOWLEDGEMENTS. ..........................

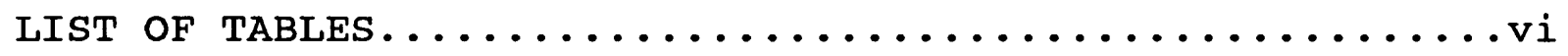

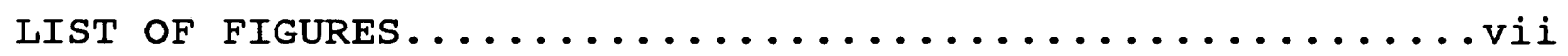

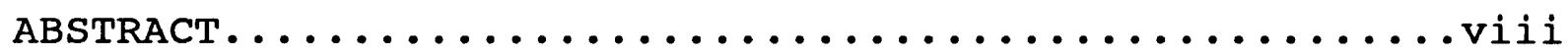

INTRODUCTION. ..............................

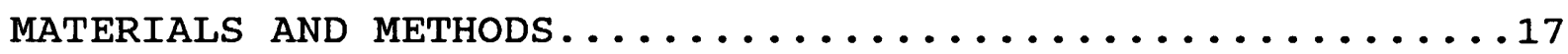

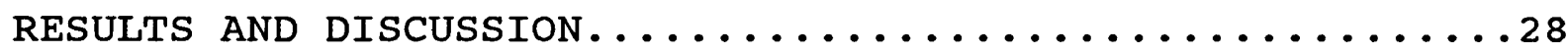

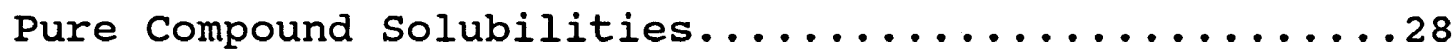

"Ideal" Solvent Solubilities........................

Benzene/Water Interactions.....................

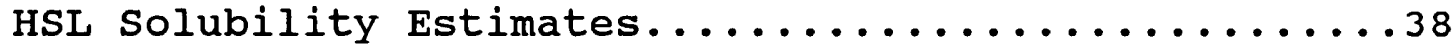

Binary Mixture Experiments................48

Multicomponent Mixture Experiments.............59

Solid Solubility Kinetics..................62

Creosote Experiment.....................65

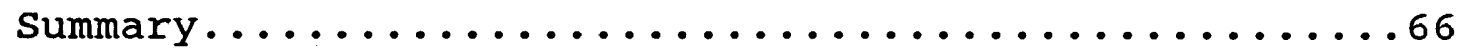

CONCLUSIONS............................. 67

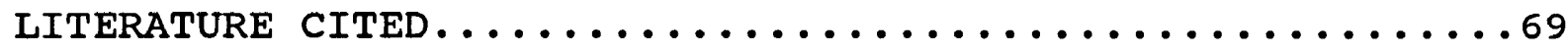

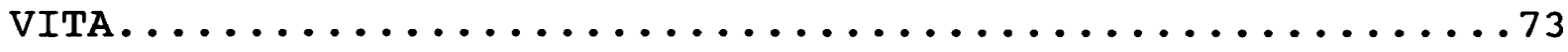




\section{ACKNOWLEDGEMENTS}

I would like to thank my committee for their reassuring support, understanding, and guidance, especially Dr. Robert J. Huggett, Dr. Robert C. Hale, and Dr. Richard L. Wetzel. And Dr. William G. MacIntyre, my major professor, for his constant prodding and direction to keep me confident and directed throughout the course of the project. For the insight to the world of geology and the rainy day drilling wells, I thank Dr. Gerald H. Johnson. And without whom, the project could not have been accomplished I would like to thank Dr. David R. Burris, and the USAF Engineering and Services center Laboratory for the financial support and the use of their facilities during my stay.

The successful completion of the project was dependent on the emotional support and distraction afforded by my fellow chemistry students Laurence Libelo, Kitty Gallagher, Jennie Gundersen, and Robbie Mothershead. And Phyllis Howard and Ellen Harvey for the administrative and technical expertise I relied heavily on while in Florida. Dr. Thomas stauffer, Chris Antworth, and Mike Henley for the technical support and friendship in Florida while I was away from home. And the rest of the people at VIMS and the endurable Bubbas soccer team for always being ready with a new perspective when the going got slow, thanks again. 


\section{IIST OF TABLES}

Table

Page

1. Aqueous Solubility of polynuclear aromatic hydrocarbons at $25 \pm 0.3^{\circ} \mathrm{C} \ldots \ldots \ldots \ldots \ldots \ldots . \ldots \ldots \ldots$

2. Solubility of polynuclear aromatic hydrocarbons in benzene at $25 \pm 0^{\circ} \mathrm{C}$ examined in this study and in

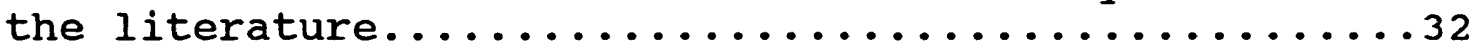

3. Solubility of PAHs in benzene and in benzene equilibrated with water at $25^{\circ} \mathrm{C} \ldots \ldots \ldots \ldots . \ldots . \ldots . \ldots 37$

4. Hypothetical supercooled liquid solubility estimates for eleven PAHs at $25^{\circ} \mathrm{C} \ldots \ldots \ldots \ldots . \ldots . \ldots . \ldots 39$

5a. Solubility of naphthalene/benzene mixtures in aqueous and hydrocarbon phases at $25^{\circ} \mathrm{C} \ldots \ldots \ldots . . \ldots 49$

5b. Solubility of anthracene/benzene mixtures in aqueous and hydrocarbon phases at $25^{\circ} \mathrm{C} \ldots \ldots \ldots \ldots . \ldots . . .49$

5c. Solubility of pyrene/benzene mixtures in aqueous and hydrocarbon phases at $25^{\circ} \mathrm{C} \ldots \ldots \ldots \ldots \ldots . \ldots . \ldots . \ldots$

5d. Solubility of phenanthrene/benzene mixtures in aqueous and hydrocarbon phases at $25^{\circ} \mathrm{C} \ldots \ldots \ldots . . \ldots 49$

6. Comparison of predicted aqueous solubilities for the Ratio method using $\gamma_{\mathrm{i}(\mathrm{hc})}=1$ and $\gamma_{\mathrm{i}(\mathrm{hc}) \text { UNIFAC }} \ldots 57$

7. Experimental and predicted aqueous phase concentration results for a seven-component hydrocarbon mixture.......................60

8. Experimental and predicted aqueous phase concentration results for a eight-component hydrocarbon mixture.......................61

9. Solubility of selected PAHs in hydddrocarbon phases equilibrated with water containing solids....64 


\section{IIST OF FIGURES}

Figure

Page

1. Diagram for the Determination of PAH "Ideal" Solvent Solubilities in Benzene.................... 19

2. Diagram for the Determination of Pure compound Solubilities in water...................... 22

3. Vessels for the Equilibration of Hydrocarbon

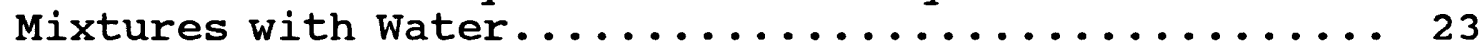

4. Comparison of Melting Point Correction and Ratio HSL solubility estimates.................... 41

5. Comparison of UNIFAC and Ratio HSL solubility estimates.............................42

6. Comparison of UNIFAC and Melting Point Correction HSL solubility estimates.................... 43

7. Comparison of Corrected UNIFAC and Ratio HSL

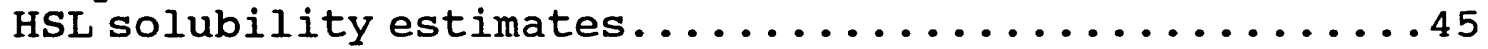

8. Comparison of Corrected UNIFAC and UNIFAC HSL solubilityestimates......................46

9. Experimentally determined aqueous phase concentrations for naphthalene/benzene and water

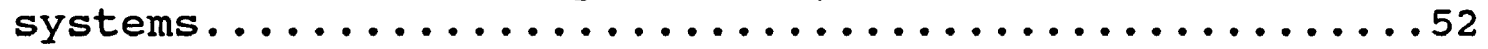

10. Experimentally determined aqueous phase concentrations for anthracene/benzene and water

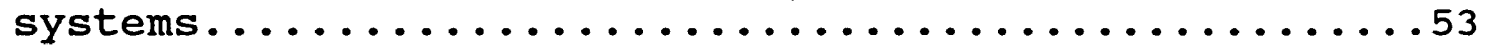

11. Experimentally determined aqueous phase concentrations for pyrene/benzene and water systems................................ 54

12. Experimentally determined aqueous phase concentrations for phenanthrene/benzene and water

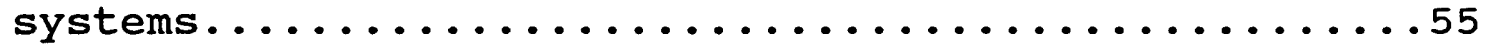




\section{ABSTRACT}

Three hypothetical supercooled liquid (HSL) solubility estimation methods are evaluated for eleven solid polynuclear aromatic hydrocarbons (PAHs). The HSL solubility is required to estimate the aqueous solubility of solids such as PAHs when they are part of a liquid hydrocarbon mixture. Aqueous solubility experimental data for binary and multicomponent liquid hydrocarbon mixtures containing PAHs were used to evaluate the HSL solubility estimation methods. A HSL solubility estimation method requiring only the component's pure compound solubility and melting point proved to be adequate for most environmental applications. 
AQUEOUS SOLUBILITY OF LIQUID HYDROCARBON MIXTURES WHICH CONTAIN DISSOLVED SOLID COMPONENTS 


\section{INTRODUCTION}

The presence of hydrocarbon mixtures in the aquatic environment requires methods to assess their potential impact. Determination of the aqueous solubility of the components in the mixture provides information which can be used in other partitioning correlations. Relationships between octanol - water partitioning and aqueous solubility have been empirically modelled (Chiou et al, 1977, Banerjee et al, 1980, Tewari et al, 1982, Yalkowsky et al, 1983, and Miller et al, 1985). These partition coefficients can be used to calculate bioaccumulation factors, toxic effects, and transport in assessing the fate of hydrocarbons in the environment (Neely et al, 1974 and Kenaga and Goring, 1980).

Liquid hydrocarbon mixtures (e.g., jet fuel, diesel fuel, crude oil, creosote) contain dissolved solids. Weathering of these mixtures increases the proportion of the dissolved solids to the total, thus the importance of assessing the fate of these compounds in the environment increases. These weathered liquid hydrocarbon mixtures are typified for example by the creosote lenses found in the Elizabeth River (Lu, 1982) and tar balls from crude oil spills.

In order to assess the fate of such a mixture in the 
aquatic environment, it is essential to be able to predict the aqueous solubility of these higher melting point components, as well as the liquid components. The hypothetical supercooled liquid (HSL) solubility of these components can be estimated from solid component solubility and some reasonable assumptions regarding differences in energy and entropy of the solid and liquid states of the component. Assumptions used in predicting these values for the energy are based on the crystalline compound's melting behavior and the changes which occur in its entropy of fusion as it becomes a liquid (Yalkowsky, 1979). The HSL solubility can then be used to predict the water solubility of hydrocarbon mixtures with components that are solid at environmental temperatures. These HSL solubilities of the higher melting point, solid components can be treated as liquid solubilities for use in predicting the solubilities of hydrocarbon mixtures in water. This chemical thermodynamic hypothetical or theoretical HSL state, which in reality can not exist, permits solid components of liquid mixtures to be treated as if they were liquids. Since the solid components of liquid hydrocarbon mixtures can then be handled as liquids, the aqueous solubility behavior of those mixtures can be predicted (Leinonen and MacKay, 1973, Leinonen, 1976, Banerjee, 1984, Burris and MacIntyre, 1985, $1986,1987)$. Then the correlations of aqueous solubility to partition coefficient which have been established (Banerjee 
et al, 1980, Tewari et al, 1982, Yalkowsky et al, 1983, and Miller et al, 1985) can be used. Although the chemical thermodynamic approximations involved in HSL solubility have been described (Hildebrand and Scott, 1962), experimental validation of the HSL solubility prediction methods has not been accomplished.

In the work reported here, several methods of estimating hydrocarbon mixtures solubilities are evaluated. These methods are compared, and predictions evaluated against data from aqueous solubility experiments with liquid hydrocarbon mixtures containing solid components at system temperature.

Experimental determinations of the equilibrium aqueous solubilities of these liquid hydrocarbon mixtures were done in the laboratory. These conditions are unrealistic due to the non-equilibrium conditions and the mass transfer limitations relative to the situation expected to occur with hydrocarbon mixtures released into a shallow aquatic environment like the Elizabeth River. But it is necessary to first test the methods of HSL solubility estimation under controlled equilibrium conditions before attempting to include the effects of dynamic flow variables, and transport and reaction kinetics of a real estuary. The ability to predict the actual aqueous concentrations of the solid components of these liquid hydrocarbon mixtures in dynamic aquatic environments could be a future goal, but can only be 
attained after the equilibrium behavior associated with these situations is known.

The thermodynamic study of ideal mixtures of pure liquids in solution at equilibrium requires the use of the partial molar free energy or the chemical potential of the system. The chemical potential $\left(\mu_{i}\right)$ of a component $i$ in solution then varies with the activity $\left(a_{i}\right)$ of the component i as:

$$
\mu_{i}=\mu_{i}^{*}+R T \ln a_{i}
$$

Where superscript * indicates the reference state for the component when the activity of component $i, a_{i}$ is unity, $R$ is the ideal gas constant, and $T$ is the temperature of the system at constant pressure. The activity of a component is the product of the component mole fraction, $x_{i}$ and its activity coefficient, $\gamma_{i}$ in equation 2 .

$$
a_{i}=X_{i} \gamma_{i}
$$

The solubility of a single liquid hydrocarbon at equilibrium with water is described using its chemical potentials in the hydrocarbon phase (3) and in water (4).

$$
\mu_{i(h c)}=\mu^{*}{ }_{i}+R T \ln \gamma_{i(h c)} X_{i(h c)}
$$




$$
\mu_{i(a q)}=\mu^{*}{ }_{i}+R T \ln \gamma_{i(a q)} X_{i(a q)}
$$

Where the superscript * indicates the pure hydrocarbon in its reference state. The subscripts $i(a q)$ and $i(h c)$ indicate the aqueous phase and hydrocarbon phase of the single liquid component $i$. At equilibrium the chemical potentials in each phase for the component are equal to each other, $\mu_{\mathrm{i}(\mathrm{aq})}=\mu_{\mathrm{i}(\mathrm{hc})}$. Therefore, for a pure liquid hydrocarbon in water at equilibrium the relationship below is valid, using the same reference state for component $i$ in equations (3) and (4).

$$
X_{i(a q)}=X_{i(h c)} \frac{\gamma_{i(h c)}}{\gamma_{i(a q)}}
$$

For a multicomponent liquid hydrocarbon mixture at equilibrium with water, the system is also thermodynamically described by equations (3), (4), and (5) for each component of the mixture. Solubility of a single hydrocarbon compound $i$ in equilibrium with water is denoted here as $x^{\circ}{ }_{i(a q)}$ and equation (5) becomes:

$$
X_{i(a q)}^{\circ}=X_{i(h c)}^{\circ} \frac{\gamma_{i(h c)}^{\circ}}{\gamma_{i(a q)}^{\circ}}
$$

Division of $\mathrm{X}_{\mathrm{i}(\mathrm{aq})}$ from equation (5) for the concentration of a 
single liquid hydrocarbon in a multicomponent mixture dissolved in water by the concentration of a pure liquid hydrocarbon dissolved in water, $\mathrm{X}_{\mathrm{i}(\mathrm{aq})}^{\circ}$ from equation (6) gives the general relation for the dissolution of components of a mixture with water.

$$
X_{i(a q)}=\frac{X_{i(h c)} \gamma_{i(h c)} \gamma_{i(a q)}^{\circ}}{X_{i(h c)}^{\circ} \gamma_{i(h c)}^{\circ} \gamma_{i(a q)}} X_{i(a q)}^{\circ}
$$

Assuming negligible water enters the pure hydrocarbon phase during equilibration then there are negligible component interactions in the pure hydrocarbon phase, $\mathrm{x}_{\mathrm{i} \text { (hc) }}$ and $\gamma_{\mathrm{i}(\mathrm{hc})}$ become unity (i.e. 1). If there are no interactions between the hydrocarbon components in the aqueous phase, then $\gamma_{i \text { (aq) }}^{\circ}$ and $\gamma_{i(a q)}$ are equal and equation (7) becomes equation (8) which describes the aqueous solubility of a liquid hydrocarbon in a multicomponent mixture equilibrated with water at system temperature and constant pressure.

$$
X_{i(a q)}=X_{i(h c)} \gamma_{i(h c)} X^{0}{ }_{i(a q)}
$$

This equation for aqueous solubility of a single component in a liquid hydrocarbon mixture, equation 8 has been evaluated by a number of researchers (Leinonen and Mackay, 1973, Burris and MacIntyre, 1985, Banerjee, 1985). Using concentration $(\mathrm{mg} / \mathrm{L})$ terms in place of mole fraction units for the aqueous phase, equation 8 becomes equation 9 . 


$$
C_{i(a q)}=X_{i(h c)} C_{i(a g)} \gamma_{i(h c)}
$$

Where $C_{i(a q)}$ and $C^{\circ}{ }_{i(a q)}$ are the aqueous solubility of component $i$ as part of a mixture and as a pure compound, respectively; $\mathrm{X}_{\mathrm{i}(\mathrm{hc})}$ is the mole fraction of component $i$ in the hydrocarbon mixture and $\gamma_{i(\mathrm{hc})}$ is the activity coefficient of the component $i$ in the hydrocarbon mixture.

If pure component $i$ is a solid at system temperature, the HSL solubility of component $i$ must be used for that component in equation (9) in place of $\mathrm{C}^{\circ}{ }_{\mathrm{i}(\mathrm{aq})}$, the solubility of pure component $i$ in water. The HSL solubility of a component which is solid at the system temperature differs from the pure compound solubility by an amount related to the energy required in the transition from a solid to a liquid. The HSL solubility is essentially the pure compound solubility of the solid plus the solid - liquid transition energy (heat), hypothesizing the imaginary existence of the solid as a liquid in its pure state at the system temperature. The HSL solubility can be estimated in several ways, three of which are described below.

Yalkowsky and Valvani, 1980 describe the pure compound solubility of a single crystalline compound in water as two approximately separable controlling factors. The first is a liquid - liquid interaction (liquid hydrocarbon - water) factor which describes the activity of the crystalline 
compound in water as if it were a liquid. And the second is a crystal interaction (crystal to a liquid) factor which describes the crystalline compound's transition into a liquid. These two interactions are related in the following equation (Yalkowsky and Valvani, 1980).

$$
\log C_{i(a q)}^{\circ}=\log X_{i(I)}^{\circ}-\log \gamma_{i(a q)}^{\circ}
$$

Here, $\mathrm{C}^{\circ}{ }_{\mathrm{i}(\mathrm{aq})}$ is the pure compound solubility of the crystal in water, $\mathrm{x}^{\circ}{ }_{\mathrm{i}(\mathrm{l})}$ in mole fraction units is the crystal interaction term or the crystalline component i solubility in an "ideal" solvent, and $\gamma^{\circ}{ }_{\mathrm{i}(\mathrm{aq})}$ is the activity coefficient in water of the solid assuming it behaves as a liquid, or the liquid interaction term from which the HSL solubility is derived. These two terms govern the solubility behavior of a single solid compound in water.

The crystal interaction term, $\mathrm{x}^{\circ}{ }_{\mathrm{i}(\mathrm{l})}$ from equation 10 is described by its activity. The activity of a crystalline solid compound dissolved in an ideal solution is governed by the thermodynamic relationship (Hildebrand \& Scott, 1962).

$$
\ln a^{s}=\frac{-\Delta H_{f}\left(T_{m}-T\right)}{R T_{m} T}+\frac{\Delta C_{p}\left(T_{m}-T\right)}{R T}-\frac{\Delta C_{p}}{R} \ln \frac{T_{m}}{T}
$$

Where $a^{s}$ is the activity of the solid in an ideal solution, 
$\Delta \mathrm{H}_{\mathrm{f}}$ is the heat of fusion at the melting point, $\mathrm{R}$ is the gas constant, $T_{m}$ is the melting point temperature, $T$ is the temperature of the solution, and $\Delta C_{p}$ is the heat capacity difference between the liquid and the solid states. The terms required in the above equation to obtain $a^{s}$ are not readily accessible for most compounds, particularly the heat capacity difference, $\Delta c_{p}$. In a strictly ideal solution the activity $\left(a^{s}\right)$ of the compound in the ideal solution is equal to its mole fraction $\mathrm{x}_{\mathrm{i}(\mathrm{l})}^{\circ}$ in the ideal solution over the entire composition range and over a nonzero range of temperature and pressure (Hildebrand and Scott, 1962 and Yalkowsky and Valvani, 1980).

$$
a^{s}=X_{i(I)}^{0}
$$

Assuming $\Delta C_{p}$ is quite small and $\left(T_{m}-T\right) / T$ is approximately equal to in $\left(\mathrm{T}_{\mathrm{m}} / \mathrm{T}\right)$, then the two terms containing $\Delta \mathrm{C}_{\mathrm{p}}$ are equal and equation 11 is approximated by the relationship (Yalkowsky \& Valvani, 1980).

$$
\log X_{i(I)}^{\circ} \approx \frac{-\Delta H_{f}\left(T_{m}-T\right)}{2.303 T_{m} T}
$$

Now, $\Delta G_{f}=\Delta H_{f}-T_{m} \Delta S_{f}$, and at the melting point of the compound the Gibbs free energy change $\left(\Delta G_{f}\right)$ is zero by definition, so the heat of fusion, $\Delta H_{f}$ equals $T_{m} \Delta S_{f}$. At room temperature, $25^{\circ} \mathrm{C}\left(298^{\circ} \mathrm{K}\right)$, and taking the gas constant as 
$1.9872 \mathrm{cal}^{\circ} \mathrm{K}^{-1} \mathrm{~mol}^{-1}$ and equation 13 reduces to:

$$
\log X_{i(I)}^{\circ}=\frac{-\Delta S_{f}}{1364}\left(T_{m}-T\right)
$$

This relationship approximates the mole fraction solubility of a crystal solute in an "ideal" solvent, the crystal interaction term for a single compound from equation 10. An "ideal" solvent is one which is similar in polarity and intermolecular attraction to the selected solute. For higher molecular weight aromatic hydrocarbons, benzene is used as an "ideal" solvent (Yalkowsky and Valvani, 1980). To determine the liquid interaction term from equation 10 , the equation for a single liquid hydrocarbon in a mixture with water, equation 15 is used:

$$
X_{i(a q)}^{\circ}=X_{i(h c)}^{\circ} \frac{\gamma_{i(h c)}^{\circ}}{\gamma_{i(a q)}^{\circ}}
$$

Assuming no water enters the hydrocarbon phase then $\mathrm{X}^{\circ}{ }_{\mathrm{i}(\mathrm{hc})}$ and $\gamma_{\mathrm{i}(\mathrm{hc})}$ are unity (i.e. equal to 1 ). Ideally, a liquid hydrocarbon in water behaves in the manner of equation 16:

$$
X_{i(a q)}^{\circ}=\frac{1}{\gamma_{i(a q)}^{\circ}}
$$

or using logarithms equation 17 , 


$$
\log X_{i(a q)}^{\circ}=-\log \gamma_{i(a q)}^{\circ}
$$

We have defined the liquid interaction and crystal interaction terms of equation 10 using the ideal states of a liquid hydrocarbon in water and a solid hydrocarbon in an "ideal" solvent. Thus the pure compound solubility of a solid PAH in water, $\log \mathrm{C}_{\mathrm{i}(\mathrm{aq})}^{\circ}$ can now be predicted using equation 10.

$$
\log C_{i(a q)}=\log X^{\circ}{ }_{i(I)}-\log \gamma^{\circ}{ }_{i(a q)}
$$

The pure compound solubility in water from equation 10 is the $\log \mathrm{C}_{\mathrm{i}(\mathrm{aq})}$ for a single component $i$, it is equal to the $\log \mathrm{x}_{\mathrm{i}(\mathrm{f})}^{\circ}$, the solid component solubility in an "ideal" solvent, plus the solubility of that solid hydrocarbon component in water if it behaved as if it were a liquid in water, $\log \mathrm{X}_{\mathrm{i}(\mathrm{aq})}^{\circ}$ from equation 17 . Equation 10 is then modified below:

$$
\log C_{i(a q)}^{\circ}=\log X_{i(I)}^{0}+\log X_{i(a q)}^{\circ}
$$

The liquid interaction term, $\log \mathrm{x}_{\mathrm{i}(\mathrm{aq})}^{\circ}$ above is the hypothetical supercooled liquid (HSL) solubility state of the solid which is described by the pure solid's aqueous and "ideal" solvent solubilities. In concentration units $(\mathrm{mg} / \mathrm{L})$, this is the $\log \mathrm{C}^{(\text {(hs) }}{ }_{\mathrm{i}(\mathrm{aq})}$. Rearranging equation 18 
with concentration units, this is the first method of determining the HSL solubility.

$$
\log C_{i(a q)}{ }^{(h s 1)}=\log C_{i(a q)}^{\circ}-\log X_{i(I)}^{\circ}
$$

Which simplifies to:

$$
C^{\circ(h s l)}{ }_{i(a q)}=\frac{C_{i(a q)}^{\circ}}{X_{i(I)}^{\circ}}
$$

$\mathrm{C}^{\circ}{ }_{\mathrm{i}(\mathrm{aq})}$ and $\mathrm{X}_{\mathrm{i}(\mathrm{I})}^{\circ}$ need to be known to estimate $\mathrm{C}^{\circ(\mathrm{hss})} \mathrm{i(aq)}^{\cdot} \mathrm{X}^{\circ}{ }_{\mathrm{i}(\mathrm{l})}$ values are generally not available in the literature, so they would have to be experimentally determined. $\mathrm{C}_{\mathrm{i}(\mathrm{aq})}$ values are generally available in the literature.

A simpler method for estimating the HSL solubility from equation 19 uses an approximation for the "ideal" solvent solubility of the solid PAH. An average entropy of fusion value can be used in equation 14 (Yalkowsky, 1979, Yalkowsky et al, 1983 and Banerjee, 1985). Yalkowsky and Valvani, 1980 estimated that for rigid spherical molecules such as PAHs the entropy of fusion, $\Delta \mathrm{s}_{\mathrm{f}}$ is approximately equal to $13.5 \mathrm{cal}^{\circ} \mathrm{K}^{-1} \mathrm{~mol}^{-1}$. Using this value in equation 14 and converting from Kelvin to centigrade at a system temperature of $25^{\circ} \mathrm{C}$, equation 14 can be simplified to: 


$$
\log X_{i(I)}=-0.01\left(T_{m}-25\right)
$$

The substitution of the value for $\log \mathrm{x}_{\mathrm{i}(\mathrm{I})}^{\circ}$ above into equation 19 denotes the second estimate of the HSL solubility called the melting point correction method.

$$
\log C_{i(a q)}{ }^{(h s 1)}=\log C_{i(a q)}+0.01(m p-25)
$$

An advantage of this method is that "ideal" solvent solubilities for most solid components are not found in the literature, whereas their pure compound solubilities in water and melting points are generally available.

The third method of estimating HSL solubility is to use the UNIFAC (UNIQUAC Functional Group Activity Coefficient) model (Fredenslund et al, 1975) to estimate the infinite dilution activity coefficients, $\gamma_{\mathrm{i}(\mathrm{aq})}^{\infty}$. The model is based on the group contribution concept and activity coefficients are assumed to be comprised of two parts, a combinatorial part and a residual part.

$$
\ln \gamma=\ln \gamma^{c}+\ln \gamma^{R}
$$

Superscripts $C$ and $R$ represent the respective parts of the UNIFAC-derived infinite dilution activity coefficient. The combinatorial part is determined using only pure component 
properties, and the parameters are calculated from van der Waals group volume and surface areas within the molecule, i.e. sizes and shapes of the functional groups on the molecule. The residual part is based on the functional group interaction parameters evaluated from experimentally determined phase equilibria data i.e. group areas and interactions. For the purposes of this study the version of UNIFAC used contained the VLE - LLE (vapor - liquid equilibria and liquid - liquid equilibria) parameter database (Gmehling et $\underline{\text { al }}, 1982$ ). Once the infinite dilution activity coefficient is determined then the mole fraction solubility as HSL solubility can be calculated as its reciprocal using the relationship described in Equation 16 . This method of estimating the HSL solubility will be called the UNIFAC method.

$$
C_{i(a q)}^{O h s I)}=(55.4 \mathrm{~mol} / L)(1000)(\mathrm{mw})\left(\frac{1}{\gamma \infty_{i(a q) U N I F A C}}\right)
$$

Where $55.4 \mathrm{~mol} / \mathrm{L}$, molecular weight (mw), and 1000 convert the reciprocal of the UNIFAC-derived $\gamma_{\mathrm{i}(\mathrm{aq})}^{\infty}$ to concentration units. The advantage of this method is that only a computer, the UNIFAC software, and a knowledge of the compound's chemical structure are necessary to determine the HSL solubility of a component in a liquid hydrocarbon mixture.

The aqueous solubility of liquid hydrocarbon mixtures 
containing solid components which have melting points above the system temperature are determined and used to evaluate three methods for estimating the HSL solubility. The three methods are: the ratio, equation 20 , the melting point correction, equation 22, and the UNIFAC method, equation 24 of estimating the HSL solubility are evaluated with respect to the experimental results. If an-easy-to use method of estimating HSL solubility with confidence was available it would be of use for environmental applications. 


\section{MATERIALS AND METHODS}

\section{Chemicals}

The polynuclear aromatic hydrocarbons (PAHs) with their associated purities used were anthracene (>99\%) and phenanthrene (>98\%) from Eastman Kodak; chrysene (98\%), 9methylanthracene (98\%), 2-ethylanthracene (98\%), 2,6dimethylnaphthalene (99\%), pyrene (>99\%), fluorene (98\%), fluoranthene (+98\%), 1-methylnaphthalene (99\%), and 2methylnaphthalene (98\%) from Aldrich; naphthalene (>99\%) from Fluka; and 1,1'binaphthyl (99\%) from Pfaltz and Bauer. Solvents used in the experiments were benzene ( $99.9 \%$ ) from Aldrich, pentane (98\%) from Burdick and Jackson, acetonitrile (HPLC grade) from Fisher scientific, methylene chloride (99.9\%) from J.T. Baker, and water from a Millipore Milli-Q Reagent Grade Water System with distilled water feed. Creosote was obtained from Wood Products, Inc. Warsaw, VA 22572 .

\section{Procedures}

A Varian model 3700 gas chromatograph (GC) or a Hewlett-Packard 5890A gas chromatograph (GC) with flame ionization detection and a split/splitless injector was used 
for organic phase analysis. The Varian GC contained a Supelco, Inc. SPB-5, $30 \mathrm{~m} \times 0.75 \mathrm{~mm}$ i.d. glass column with a 1.0 $\mathrm{m}$ film thickness and the Hewlett-Packard GC used a $J \& W$ Scientific DB17-30N, $30 \mathrm{~m} \times 0.25 \mathrm{~mm}$ i.d. fused silica column with a $0.25 \mu \mathrm{m}$ film thickness. Oven temperature was programmed on the Varian GC from 40 to $270^{\circ} \mathrm{C}$, and on the Hewlett-Packard GC from 50 to $270^{\circ} \mathrm{C}$ at $15^{\circ} / \mathrm{min}$. with an initial hold of 2 minutes and a final hold of 5 minutes with the injector temperature at $300^{\circ} \mathrm{C}$, and the detector temperature at $290^{\circ} \mathrm{C}$. The carrier gas was helium for both GCs with a head pressure of 8 psi on the Varian GC and 15psi on the Hewlett-Packard GC. Air and hydrogen head pressures were 28 psi and 32 psi respectively. A Hamilton Co. model $701 \mathrm{~N} 10 \mu 1$ GC syringe was used to inject standards and samples which were split 30 seconds after injection. Data was collected, integrated, and stored on a Hewlett-Packard 3357 Laboratory Data System via a Hewlett-Packard model 18652A analog/digital converter. Both Gcs produced comparable results and were used interchangeably.

A Hewlett-Packard GC-MS was used to analyze a creosote sample used in the hydrocarbon mixture - water equilibration studies that was diluted 1000 times by volume with methylene chloride.

The following procedure was used to determine the PAH solubility in benzene: An excess of a PAH was placed in a $15 \mathrm{ml}$ centrifuge tube with a teflon lined screw cap and 


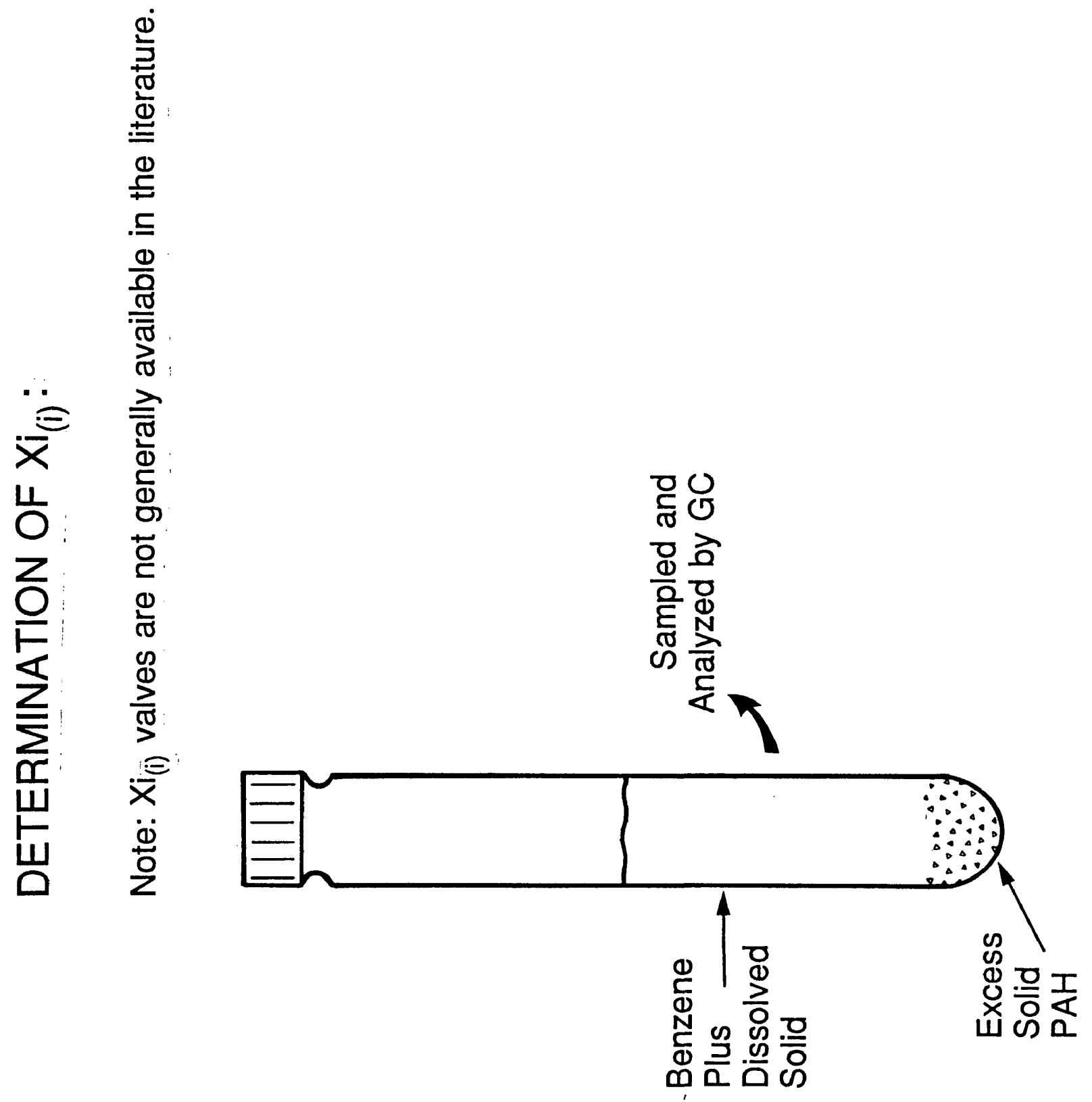


approximately $2 \mathrm{mls}$ of benzene added, Figure 1. Each tube was shaken and then incubated at $25 \pm 0.3^{\circ} \mathrm{C}$ for 48 hours. A $100 \mathrm{ul}$ aliquot of the solution was removed from the equilibrated tube and quickly added, either by syringe injection or pipetting, to a $10 \mathrm{ml}$ volumetric flask containing the 1-methylnaphthalene internal standard in pentane and the volume adjusted. The contents were mixed and analyzed by GC. Five replicates were done for each compound. Standards of the PAHs except for chrysene were prepared in pentane; chrysene was prepared in methylene chloride because of its low solubility in pentane. Solute standard mixtures were prepared, injected, and their relative response factors determined daily for each compound from three replicates prior to organic phase analysis (Grob, 1977). The internal standard was prepared daily from a stock solution that was stored in the freezer when not in use, and the stock solution remade after 30 days.

Aqueous solubilities of pure hydrocarbons were determined using a modified generator column method (May et al, 1978a, DeVoe et al, 1981, Velapoldi et al, 1983, Dickhut et al, 1986, and Billington et al, 1988). Generator columns were constructed of coiled stainless steel tubing $60 \mathrm{~cm} \mathrm{x}$ $0.4 \mathrm{~cm}$ i.d. fitted with stainless steel $2.0 \mathrm{um}$ fritted disks and zero dead volume reducing unions at each end. Columns were packed with approximately 3.5 grams of Chromosorb W (60/80 mesh from Alltech Associates) previously baked 
overnight at $350^{\circ} \mathrm{C}$ and coated with $1 \% \mathrm{w} / \mathrm{w}$ of the PAH of interest. Coating of the solid support (Chromosorb $\mathrm{W}$ ) was accomplished by dissolving a known amount of the PAH in 100 mls. of methylene chloride, mixing with a known amount of Chromosorb $\mathrm{W}$, and stripping off the solvent with a rotary evaporator (May et al, 1978a). Naphthalene and 2-methylnaphthalene have high aqueous solubilities and thus were prepared as $20 \% \mathrm{w} / \mathrm{w}$ coatings to provide adequate generator column life. Each generator column was packed under vacuum and mechanically vibrated to aid in settling.

A Waters Associates model 510 pump provided the flow of Milli-Q water through the generator columns. A $100 \mathrm{~cm}$. long coil of 1/16 inch stainless steel capillary tubing was used as a heat exchanger and pulse dampener to equilibrate the water entering the generator column. The heat exchanger and generator column were immersed in a $25 \pm 0.1^{\circ} \mathrm{C}$ Neslab RTE-10 water bath, (see Figure 2). Pump flow was set at $1.0 \mathrm{ml} / \mathrm{min}$ and checked with a volumetric flask and stopwatch for confirmation. Generator columns were flushed with approximately $100 \mathrm{mls}$ of water prior to collecting an aqueous sample. This volume of flushing was sufficient to remove more polar and thus more water soluble impurities occurring in the standards.

Vessels used for the equilibration of hydrocarbon mixtures in water were designed by Burris and MacIntyre, 1985 and are shown in Figure 3. The vessels are fitted with 

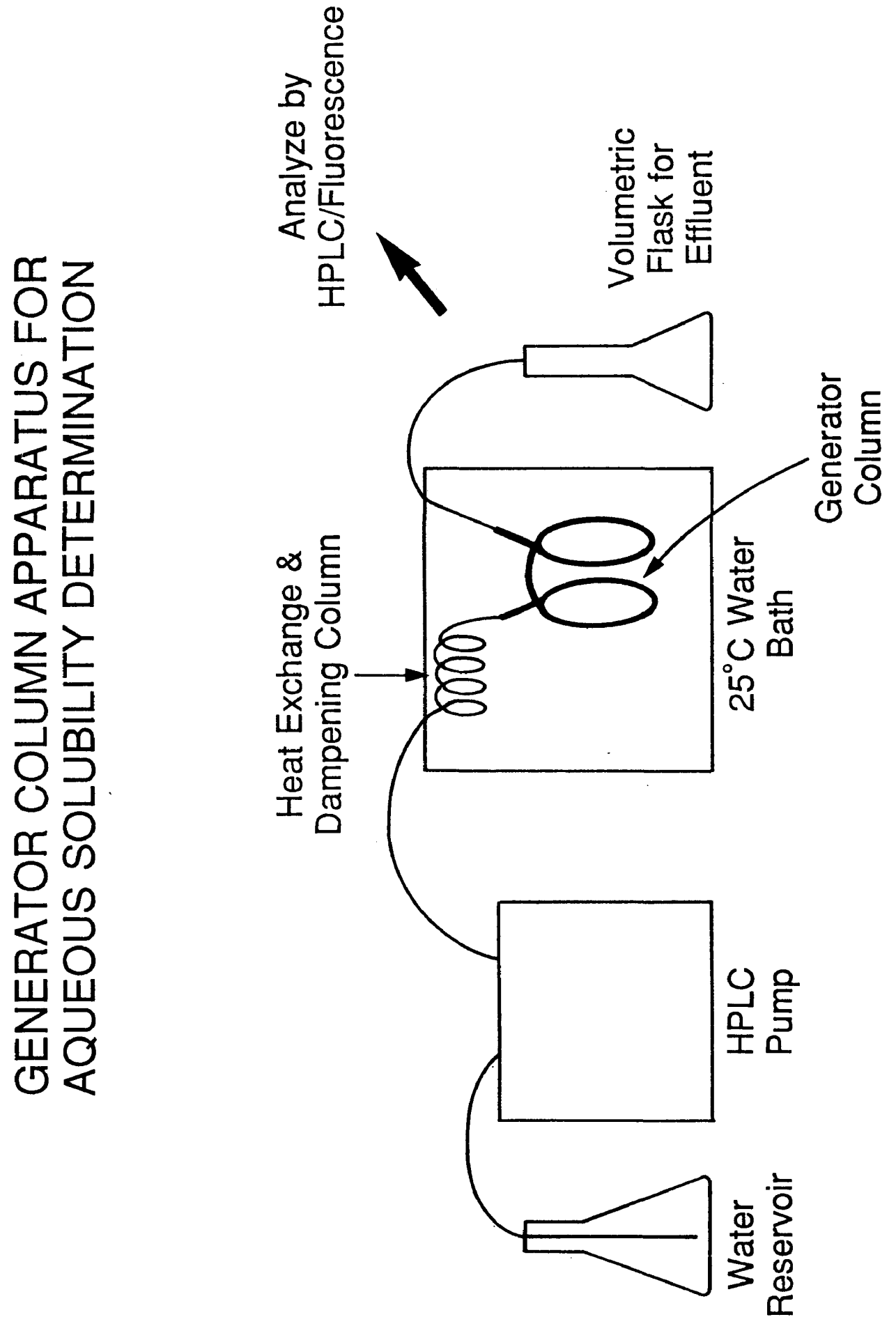


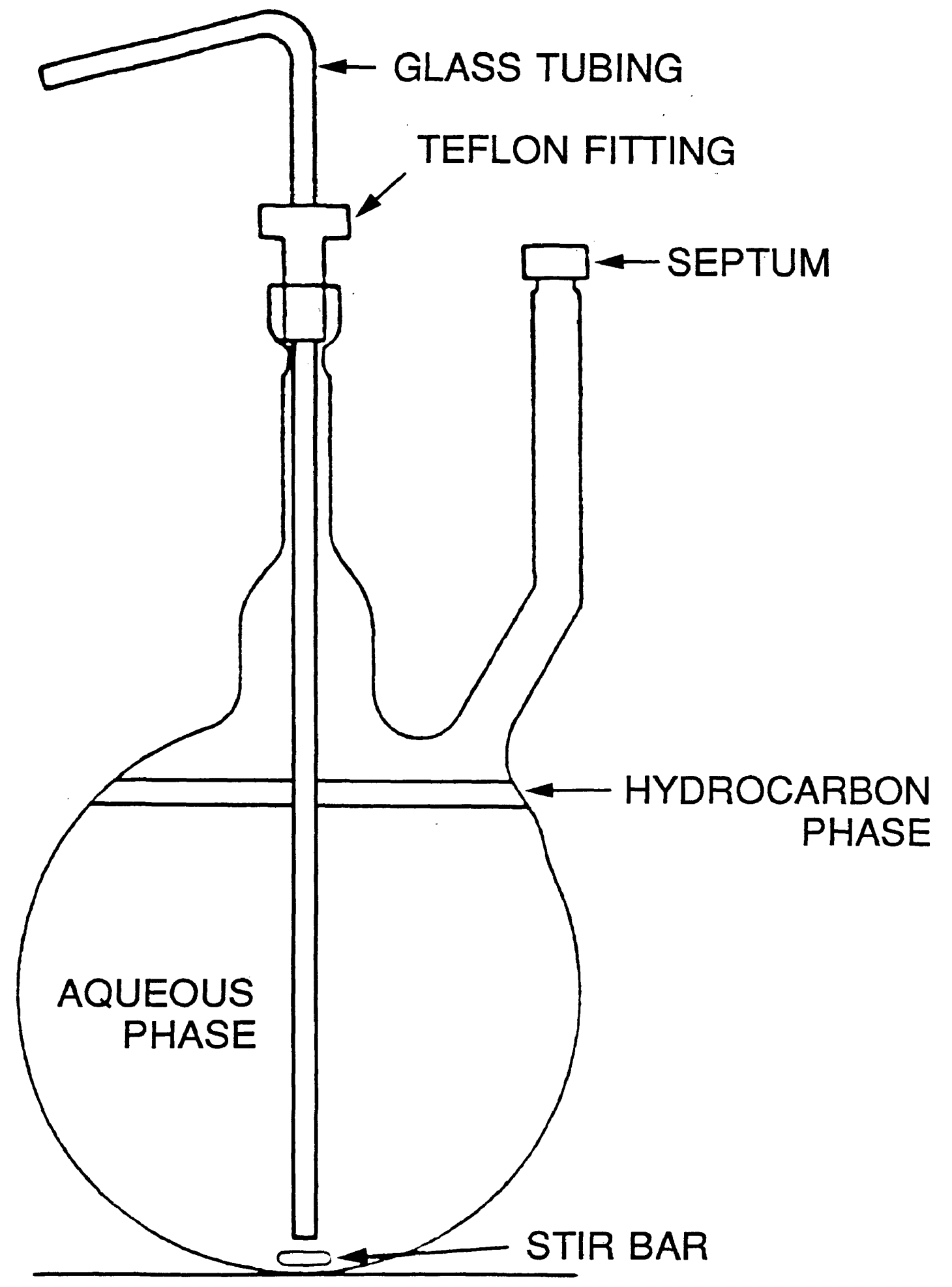


a teflon septa and screw cap on the sidearm and a teflonferrule and fitting sealing the glass tube in the main arm. In the hydrocarbon mixture equilibration experiments, $350 \mathrm{mls}$. of Milli-Q water and $5 \mathrm{mls}$ benzene were mixed on a stir plate without vortex formation for 30 minutes in a $25 \pm$ $0.2^{\circ} \mathrm{C}$ incubator. This allows vapor and water in the vessel to become saturated with benzene prior to adding the organic phase. If the water is not saturated with benzene first, the solute in the hydrocarbon phase might form a solid phase because of different rates of dissolution of the hydrocarbon mixture components and the benzene into the aqueous and vapor phases. The formation of a third, possibly metastable, solid phase would have created a much more complicated system. After initial equilibration with benzene, the remaining components of the hydrocarbon mixture were gently introduced as a liquid solution through the opened sidearm of the vessel using a 50 cc glass syringe with stainless steel Luer-lok needle. Care was taken to avoid disturbing the hydrocarbon - water interface. The flask was resealed and allowed to equilibrate for 48 hours with gentle stirring of the water phase by a magnetic stirrer. The stirrer was set on the lowest setting which provided mixing without vortex formation and thus avoided the formation of droplets of the hydrocarbon phase in the water. Flasks were visually inspected during equilibration for the presence of droplets and, if they were observed, the 
experiment was repeated. Total organic phase volume was 30 to $50 \mathrm{mls}$. After equilibration, water was sampled by injecting air into the vessel headspace through the teflon septa of the sidearm to expel the aqueous phase. A $10 \mathrm{ml}$ volume of water was expelled to flush out the glass tube and was discarded, and immediately following this a sample was collected in a $10 \mathrm{ml}$ volumetric flask containing the $0.1 \mathrm{ml}$ of the internal standard. Three to five replicates of the aqueous phase were done. Immediately after the aqueous phase sampling, the organic phase was sampled by opening the screw cap on the sidearm, tilting the vessel towards the sidearm and removing a $100 \mu l$ aliquot of the hydrocarbon phase using a pipet or a syringe. The aliquot was immediately added to a $10 \mathrm{ml}$ volumetric flask containing 0.1 ml of the 1-methylnaphthalene internal standard, the volume adjusted using pentane and then analyzed by GC as described above for the "ideal" solvent solubilities.

Analysis of the all aqueous solutions generated from the pure compound generator columns and the binary and multicomponent liquid hydrocarbon mixture - water equilibration experiments were done using a Waters Associates High Performance Liquid Chromatograph (HPLC) with a reverse phase analytical column coupled to a fluorescence detector similar to the methods used by Schwarz and Wasik (1976), Schwarz (1977) and Billington et al (1988). An aliquot of the aqueous phase was injected onto a Bondex 10 
C-18, 300mm x $3.9 \mathrm{~mm}$ analytical column through a Rheodyne model 7125 injection valve. Elution of the solutes was done using a Waters Associates Model 660 Solvent Programmer interfaced with two model 6000A Solvent Delivery Systems coupled to a Applied Biosystems (ABI) spectroflow 980 Programmable Fluorescence detector. A water/acetonitrile solvent gradient from $0 \%$ to $70 \%$ acetonitrile at 2.0 mls./min. ran in 10 minutes. The initial hold time at $100 \%$ water was 2 minutes and the final hold time was dependent on the elution of the individual solute. The rate of change during the gradient from water to acetonitrile was exponential, changing rapidly at first and then slowly levelling off at $70 \%$ acetonitrile. The monochromatic fluorescence detector was equipped with a deuterium lamp, the excitation wavelength was $265 \mathrm{~nm}$, the emission wavelength was $370 \mathrm{~nm}$ and the flow cell volume was $5 . \mu 1$. Excitation and emission wavelength settings were optimized for maximum detection of compounds with the lowest aqueous concentrations and/or minimum fluorescence response, similar to HPLC/fluorescence analysis by Schwarz and Wasik (1976) and Schwarz (1977). Also, injection volume varied from 20 $1000 \mu 1$ depending upon the aqueous solubility and the fluorescence intensity of the particular solute. Standards for the individual solutes were prepared and analyzed daily to determine the relative response factors. Stock solutions of each $\mathrm{PAH}$ in acetonitrile were prepared in pyrex 
volumetric flasks with ground glass stoppers, and were sealed with teflon tape and kept in a freezer when not in use. Daily standards were made from stock solutions by adding an aliquot added to a $100 \mathrm{ml}$ volumetric flask containing the 1-methylnaphthalene internal standard in acetonitrile, and the volume adjusted to $100 \mathrm{mls}$ with MilliQue water. Fluorescence detector output data was collected, integrated, and analyzed using the Hewlett-Packard 3357 Laboratory Data system. 


\section{RESULTS AND DISCUSSION}

\section{Pure Compound Solubilities}

Aqueous solubilities of the eleven PAH compounds determined by analysis of effluents from generator columns are shown in Table 1 . These aqueous solubility results are in good agreement with the literature values from various sources. Although the values for the aqueous solubility of polynuclear aromatic hydrocarbons are well documented in the literature, determination of their aqueous solubility was repeated here. This was to establish the techniques to be used during binary and multicomponent mixture analysis, and establish confidence in the accuracy and precision of values obtained in those determinations. Coefficients of variations for one standard deviation of the analyzed aqueous solubilities ranged from 2 - 13 percent indicating reasonably precise measurements similar to those of other authors using generator column techniques (Dickhut et al, 1986). However, disparities were found 9-methylanthracene and 2,6-dimethylnaphthalene that are perhaps worthy of further investigations. Direct analysis by HPLC with fluorescence detection permitted rapid analysis of small volumes of aqueous phase. This was essential when analyzing 
Table 1. Aqueous solubility (mg/L, $x \pm 1$ s.d.) of polynuclear aromatic hydrocarbons at $25.0 \pm 0.3^{\circ} \mathrm{C}$

\begin{tabular}{|c|c|c|c|}
\hline Compound & $\begin{array}{l}\text { Solubility } \\
\text { (this work) }\end{array}$ & $\begin{array}{l}\text { Solubility } \\
\text { (literature) }\end{array}$ & $\underline{\text { Reference }}$ \\
\hline $\begin{array}{l}2 \text {-methyl } \\
\text { naphthalene }\end{array}$ & $27.3 \pm 1.7$ & $\begin{array}{l}25.4 \\
24.6\end{array}$ & $\begin{array}{l}1 \\
2\end{array}$ \\
\hline $\begin{array}{l}\text { 2-ethyl } \\
\text { anthracene }\end{array}$ & $0.034 \pm 0.002$ & 0.027 & 6 \\
\hline $\begin{array}{l}2,6 \text {-dimethyl } \\
\text { naphthalene }\end{array}$ & $0.997 \pm 0.025$ & $\begin{array}{l}1.30 \\
2.00\end{array}$ & $\begin{array}{l}2 \\
1\end{array}$ \\
\hline Naphthalene & $30.6 \pm 3.2$ & $\begin{array}{l}31.7 \\
30.3 \\
31.3\end{array}$ & $\begin{array}{l}1 \\
4 \\
2\end{array}$ \\
\hline Pyrene & $0.107 \pm 0.002$ & $\begin{array}{l}0.135 \\
0.132 \\
0.129\end{array}$ & $\begin{array}{l}1 \\
5 \\
4\end{array}$ \\
\hline Anthracene & $0.058 \pm 0.001$ & $\begin{array}{l}0.073 \\
0.041 \\
0.075\end{array}$ & $\begin{array}{l}1 \\
4 \\
3\end{array}$ \\
\hline Chrysene & $0.0016 \pm 0.0002$ & $\begin{array}{l}0.0020 \\
0.0018\end{array}$ & $\begin{array}{l}1 \\
5\end{array}$ \\
\hline Phenanthrene & $1.00 \pm 0.02$ & $\begin{array}{l}1.29 \\
1.10 \\
1.00\end{array}$ & $\begin{array}{l}1 \\
6 \\
5\end{array}$ \\
\hline $\begin{array}{l}\text { 9-methyl } \\
\text { anthracene }\end{array}$ & $0.530 \pm 0.070$ & 0.261 & 1 \\
\hline Fluoranthene & $0.177 \pm 0.006$ & $\begin{array}{l}0.26 \\
0.206\end{array}$ & $\begin{array}{l}1 \\
5\end{array}$ \\
\hline Fluorene & $2.23 \pm 0.07$ & $\begin{array}{l}1.98 \\
1.685 \\
1.89\end{array}$ & $\begin{array}{l}1 \\
5 \\
3\end{array}$ \\
\hline $\begin{array}{l}1 \text { - Mackay and } \\
2 \text { - Eganhouse } \\
3 \text { - Wauchope a }\end{array}$ & $\begin{array}{l}\text { Shiu, } 1977 \\
\text { and Calder, } 1976 \\
\text { nd Getzen, } 1972\end{array}$ & $\begin{array}{l}4-s \\
5-N \\
6-h\end{array}$ & $\begin{array}{l}\text { Schwarz, } 1977 \\
\text { May et al, } 1978 \mathrm{~b} \\
\text { Whitehouse, } 1984\end{array}$ \\
\hline
\end{tabular}


aqueous phases in equilibration with slightly soluble binary and multicomponent mixtures, because the vessels only contained $350 \mathrm{mls}$ of water and disruption to the equilibration vessel contents needed to be minimized.

The use of generator columns for sparingly soluble hydrophobic compounds is the preferred method of aqueous analysis, as compared to batch contact or shake flask methodology (May et al, 1978b, DeVoe et al, 1981, and Billington et al, 1988). Naphthalene generator column aqueous analysis at flows of $1.0 \mathrm{ml} / \mathrm{min}$ and $3.0 \mathrm{ml} / \mathrm{min}$ for four replicates were $28.9 \pm 1.4$ and $29.5 \pm 0.7 \mathrm{mg} / \mathrm{L}$. This is consistent with May et al, (1978a) who showed that aqueous concentration was independent of flow rate over a reasonable flow range.

Elution of interfering more soluble contaminants occurred in the case of anthracene. This standard contained a small amount of phenanthrene; phenanthrene is 20 times more soluble than anthracene. The phenanthrene was easily flushed out of the anthracene generator column prior to collection of samples for anthracene aqueous solubility determinations. This observation indicates a problem in the use of generator columns containing mixtures with components varying in the degree of their aqueous solubility. Dosing toxicological experiments or measuring mixture aqueous solubilities at equilibrium would be difficult, because the 
composition of the mixture in the effluent would not be steady long enough to produce a constant dose over a useful time interval (May et al, 1978 and Velapoldi et al, 1983). Shiu et al, (1988) proposed a method of predicting the water soluble fraction of a hydrocarbon mixture (oil) using a generator column. This is essentially an oil aging experiment and would produce concentrations that might be related to the kinetic behavior of an actual environmental situation. From their experiments using generator columns, it may be possible to predict the expected aqueous solubilities of components of the mixture if the initial composition of the oil mixture, water volume, and oil volume are known. Measurement of the several variables required is difficult and the accuracy of solubility estimates is likely to be poor. In any event, all results would have to be checked by other methods.

\section{"Ideal" Solvent Solubilities}

The "ideal" solvent PAH solubilities in benzene are shown in Table 2 along with those in the literature. The "ideal" solvent solubility results do not agree for all PAH's with those of Mclaughlin and Zainal, (1959). It is believed that the GC method used in this study is more accurate than their visual method of analysis. Their method of determining the "ideal" solvent solubility of PAHs entailed sealing a tube with a known amount of solid solute 
Table 2. Solubility of polynuclear aromatic hydrocarbons in benzene (mole fraction, $x \pm 1 \mathrm{s.d.}$ ) at $25^{\circ} \mathrm{C}$ examined in this study and those of McLaughlin and Zainal, 1959.

\begin{tabular}{|c|c|c|}
\hline Compound & $\begin{array}{l}\text { Solubility } \\
\text { (this work) }\end{array}$ & $\begin{array}{c}\text { and Zainal, } \\
\text { 1959) }\end{array}$ \\
\hline $\begin{array}{l}\text { 2-methyl } \\
\text { naphthalene }\end{array}$ & $0.657 \pm 0.004$ & - \\
\hline $\begin{array}{l}2,6 \text {-dimethyl } \\
\text { naphthalene }\end{array}$ & $0.123 \pm 0.002$ & - \\
\hline $\begin{array}{l}2 \text {-ethyl } \\
\text { anthracene }\end{array}$ & $0.079 \pm 0.001$ & - \\
\hline $\begin{array}{l}\text { 9-methyl } \\
\text { anthracene }\end{array}$ & $0.317 \pm 0.017$ & - \\
\hline Anthracene & $0.024 \pm 0.001$ & $0.0103\left(35.8^{\circ} \mathrm{C}\right)$ \\
\hline Naphthalene & $0.294 \pm 0.011$ & $0.323 *$ \\
\hline Pyrene & $0.093 \pm 0.003$ & $0.059 *$ \\
\hline Chrysene & $0.0043 \pm 0.0003$ & $0.0021\left(35.6^{\circ} \mathrm{C}\right)$ \\
\hline Phenanthrene & $0.245 \pm 0.007$ & $0.191 *$ \\
\hline Fluorene & $0.162 \pm 0.004$ & $0.125 *$ \\
\hline Fluoranthene & $0.178 \pm 0.006$ & $0.120 *$ \\
\hline $\begin{array}{l}\text { - Values are } \\
\text { experiment }\end{array}$ & $\begin{array}{l}\text { ated to } 25^{\circ} \mathrm{C} \text {, } \\
\text { rations at tempe }\end{array}$ & $\begin{array}{l}\text { values are } \\
\text { re }\end{array}$ \\
\hline
\end{tabular}


and benzene, and slowly raising the temperature while rotating until the solute dissolved. At the temperature ofcomplete dissolution, the mole fraction solubility of the solute in the "ideal" solvent is known. For each PAH with a melting point under $150^{\circ} \mathrm{C}$, a series of mole fraction concentrations were determined, and the measured values extrapolated through the melting point. A solubility equation using a least squares method was developed to described the line.

$$
\log x=-m(1 / T)+C
$$

Parameters $\mathrm{m}$ and $\mathrm{C}$ are calculated for each PAH's fit to the ideal line. Using this equation, the values obtained by McLaughlin and Zainal, (1959) are extrapolated to $25^{\circ} \mathrm{C}$ and compared to those in this work, Table 2. It was found that a period of equilibration following a temperature change is needed during solute dissolution to allow transfer of heat equivalent to the heat of fusion of the crystal lattice and for the system to reach thermal equilibrium. It appears that this equilibration period may not have been of sufficient duration during heating to account for the large differences observed between the two methods. Another potential problem with their method is the requirement for visual observation of crystal dissolution, which is practical for large crystals, but for microcrystals, actual 
dissolution observations may be subject to arbitrary interpretation. GC methods for determination of "ideal" solubilities may be potentially influenced by microcrystals, but replicates did not show the wide variability of concentrations that would occur if microcrystals were sampled. "Ideal" solvent solubilities determined by equilibrating an excess of solid solute with benzene for 48 hours at $25^{\circ} \mathrm{C}$ and analyzing by GC give results which are believed to be more accurate than visual endpoint based method. Values for pyrene, phenanthrene, fluorene, and fluoranthene with higher melting points are lower by 20 to 36 percent using the visual method. Anthracene and chrysene with even higher melting points did not fit the conditions for the solubility equation, but appear to be grossly underestimated by the visual method, at least as can be extrapolated without the solubility equation. Naphthalene with a lower melting point is overestimated by extrapolation using the solubility equation as compared to the GC determined value. Still, one is led to believe that the visual method severely underestimates the "ideal" solvent solubilities of these compounds. Unfortunately, no other data on $\mathrm{PAH}$ solubilities in benzene are available with which to compare the results here and those of McLaughlin and Zainal, (1959). The precision of GC determinations of "ideal" solvent solubilities are good using the internal standard method for quantitation and relative response 
factors calculated from standards injected on a daily basis. Five replicates were done for each PAH to check for possible variations similar in magnitude to the difference between the two methods and to detect microcrystals in the aliquots sampled. Coefficient of variation for each PAH was less than 7 percent for one standard deviation indicating reasonably precise measurements. This instills confidence in the measurement techniques used, especially when dealing with compounds with low aqueous solubilities. Comparison of data with the one set in the literature provides a rough measure of accuracy even though values differed between the two methods.

\section{Benzene/Water Interactions}

A potential concern with equilibrating liquid hydrocarbon mixtures with water is the differential dissolution of the benzene and the solute into the aqueous phase, the loss of the benzene to the vapor phase, and the interaction of water entering the hydrocarbon phase. If any or all of these occurred then the possibility exists for a third, solid phase of the solute to form in the organic phase of the mixture. To check the interaction of water in the hydrocarbon phase, "ideal" solvent solubilities were compared to "ideal" solvent solubilities equilibrated with water to determine if water had an effect on the solute mole fraction of the PAH in the organic phase. The results of 
the solid, benzene, and water equilibration for three PAHs are in Table 3. Comparing the values of the $\mathrm{PAH} /$ benzene toPAH/benzene/water simultaneous experiments equilibrated at $25^{\circ} \mathrm{C}$ for 48 hours showed no significant difference between the two tests within the experimental error of the methods used. The solute concentration in the benzene pháse was not reduced due to the presence of water in the hydrocarbon phase. This leads one to believe that in these relatively small volumes and within experimental error, the activity coefficient of the solute in the hydrocarbon phase was not affected by water and is still close to unity. The magnitude of the change in the activity coefficient needed to cause solute recrystallization does not appear to come from the effects of water in the organic phase during equilibration. Water does enter the hydrocarbon phase, just not enough to make a difference.

To account for differential dissolution of the benzene and PAH solute in water and the vapor loss during equilibration, benzene was added to the equilibration vessel prior to the mixture introduction to saturate the vessel environment. This generally kept solute recrystallization from occurring upon introduction of the PAH mixture. Solute recrystallization did occurred in the phenanthrene/benzene equilibration vessel below the maximum benzene solubility in spite of these precautions. This observations leads one to believe that other factors might also be responsible (e.g., 
Table 3. Solubility of PAHs in benzene and in benzene equilibrated with water (mole fraction, $x \pm 1$ s.d.) at $25^{\circ} \mathrm{C}$

Compound

2,6 -dimethylnaphthalene

Phenanthrene

Chrysene
PAH/Benzene

$0.161 \pm 0.004$

$0.239 \pm 0.004$

$0.004 \pm 0.000$
PAH/Benzene/Water

$0.161 \pm 0.002$

$0.245 \pm 0.007$

$0.005 \pm 0.000$ 
solute surface chemistry, slow kinetics, phase boundary conditions).

\section{HSL Solubility Estimates}

The HSL solubility estimates by the three different methods are given in Table 4 . The pure compound solubilities are included in Table 4 to show that HSL solubilities can be orders of magnitude larger than pure compound solubilities. This is particularly evident for the higher melting point aromatic compounds. These compounds are less water soluble, less volatile and more strongly sorbed on sediment than the low melting point aromatic hydrocarbons. They are thus expected to remain in the sediment - water environment for long periods and potentially to have chronic toxic effects on marine organisms. Use of pure compound solubilities to predict the aqueous solubilities of solid compounds in a liquid hydrocarbon mixture released in the marine in the environment severely underestimates the aqueous phase $\mathrm{PAH}$ concentrations and consequently underestimates PAH exposure to biota.

The aqueous solubility to the "ideal" solvent solubility ratio method (using the values from Tables 1 and $2)$, equation 20, should provide the best estimate for the HSL solubility, since the main source of error would be the 


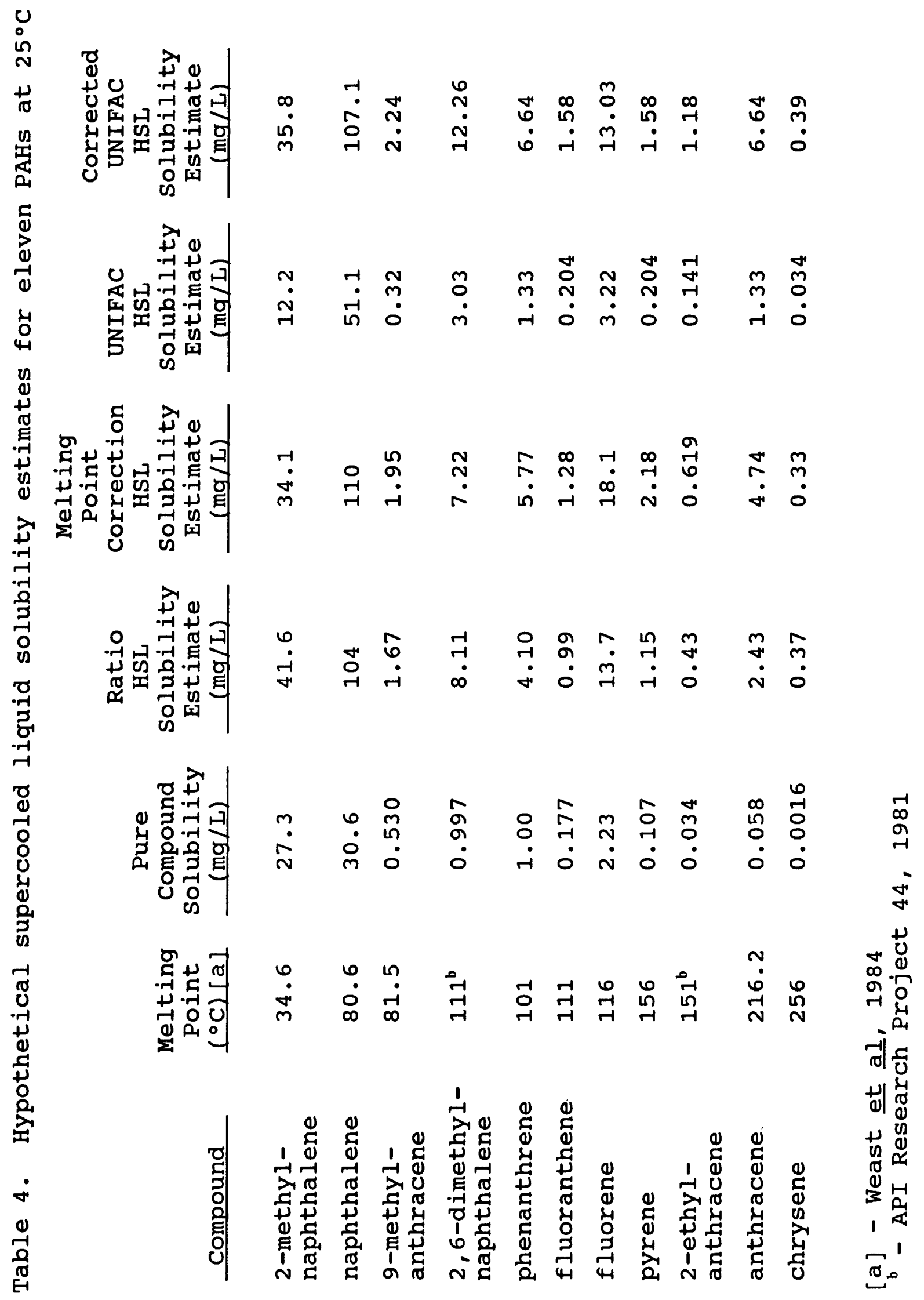


errors associated with experimental determinations. This assumes linear behavior between the hydrocarbon solute concentration and the solute aqueous concentration. The other two methods would be expected to be less accurate because of the assumptions made in deriving the melting point correction method and the empirical nature of UNIFAC. The melting point correction method results calculated from equation 22, using pure compound solubilities and melting point data from Table 3, compare well with the solubility ratio method results. As indicated in Figure 4, the estimates generally agree within a factor of two. Figure 4 is presented as a log - log plot because of the wide solubility range of the eleven PAHs. The solid line with slope equal to 1 on this figure represents perfect agreement between the methods. The agreement between these methods is encouraging, since the melting points and aqueous solubilities are generally available in the literature, making the melting point correction method relatively practical to use.

The UNIFAC method results from equation 24 do not compare as well with those of the ratio method as seen in Figure 5. If the solubility ratio method estimates are assumed to be reasonably accurate, then UNIFAC underestimates the HSL solubilities by as much as a factor of 10. Figure 6 compares UNIFAC and melting point correction method results and also indicates that the UNIFAC 

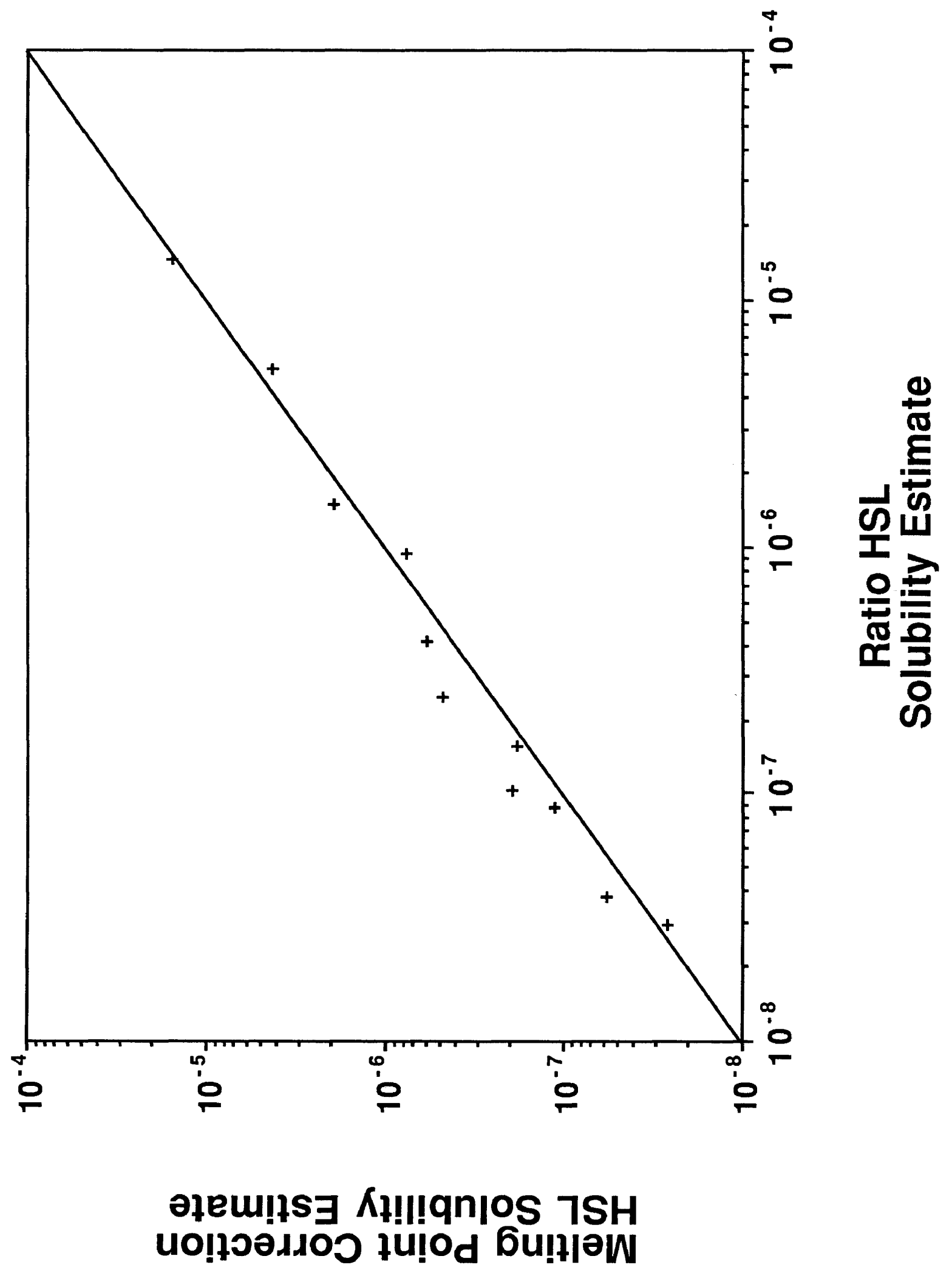


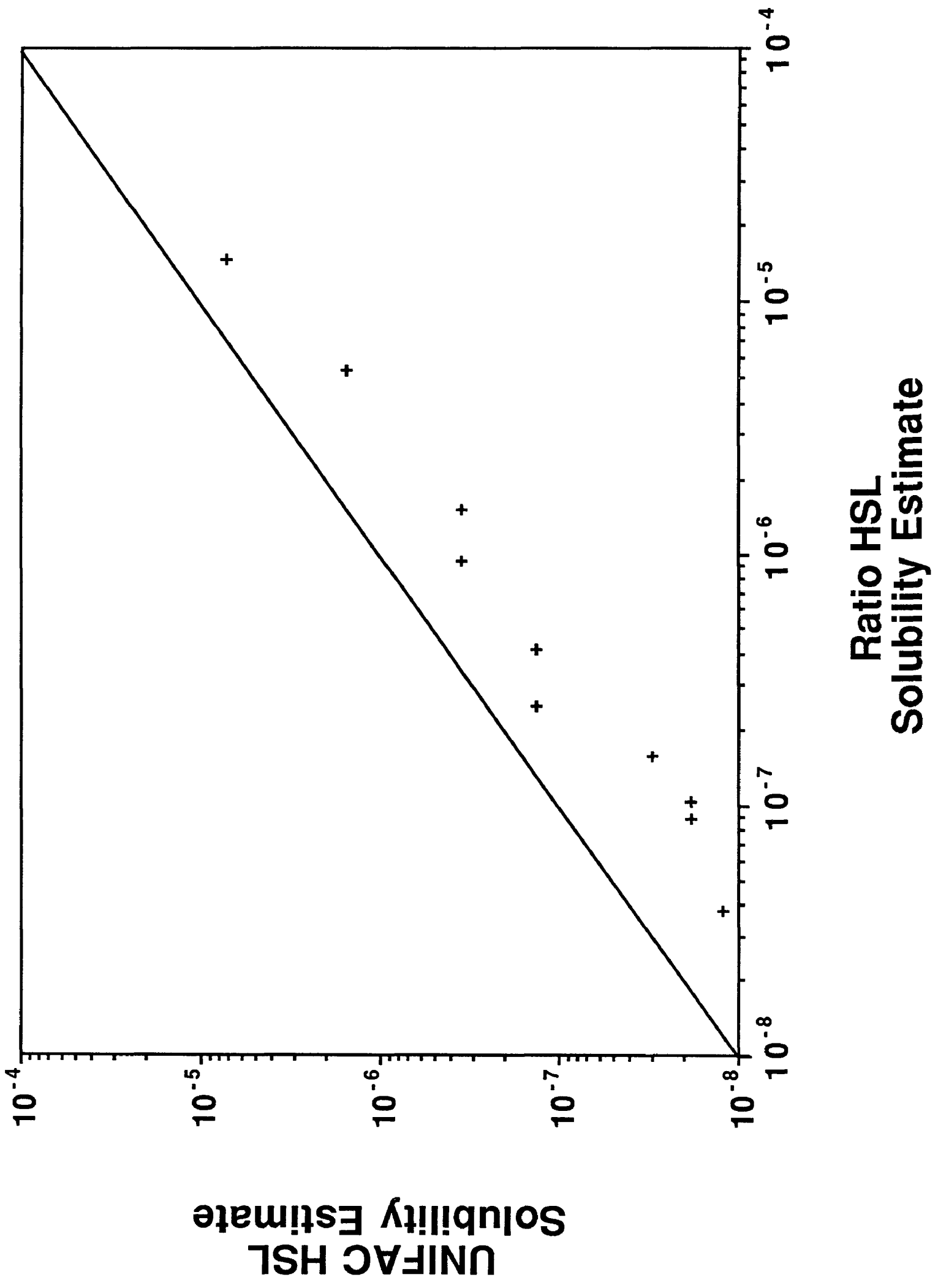




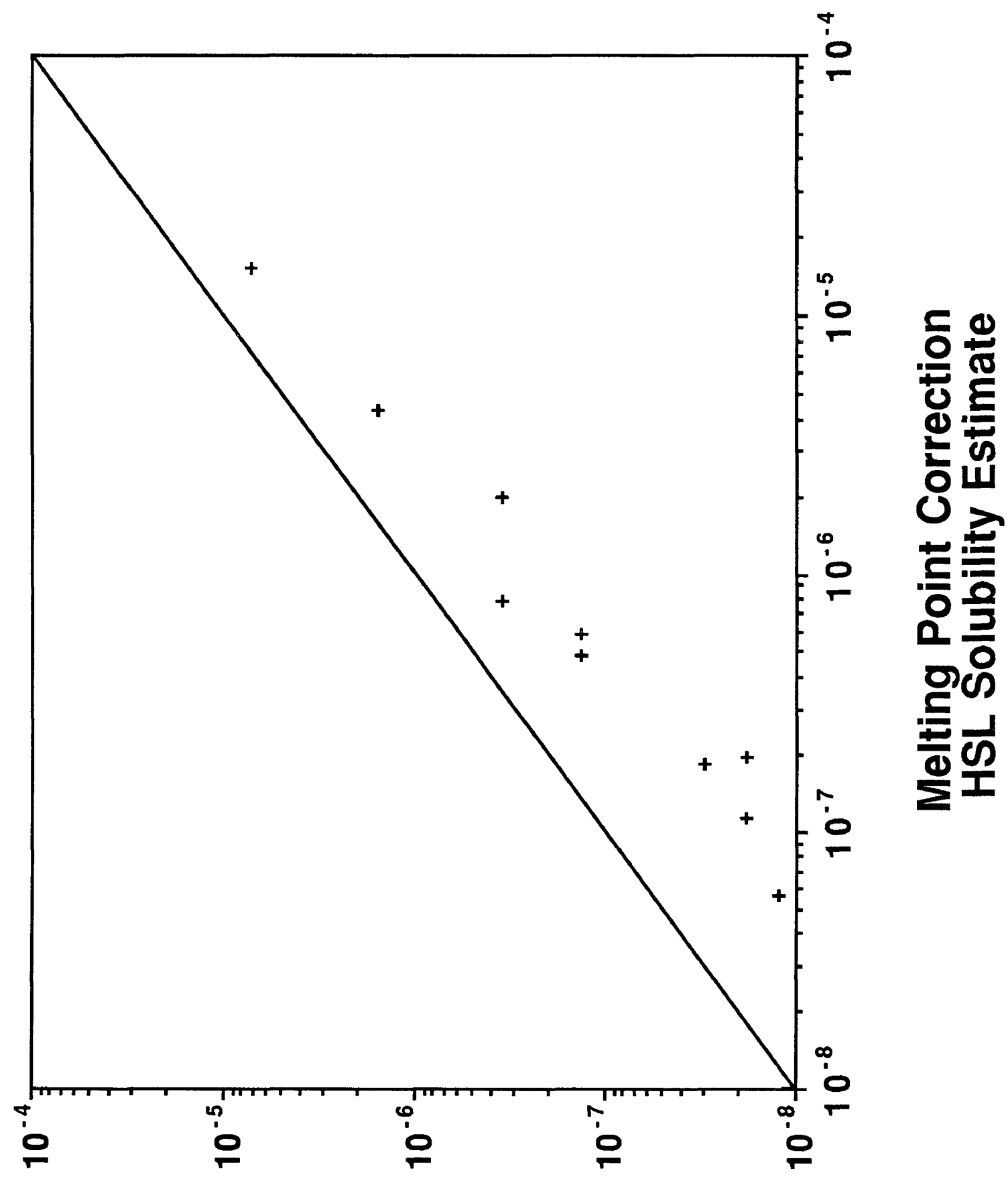

\section{әреu!!s Kł!!!qnjos

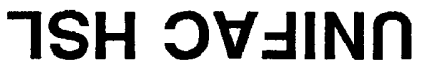


method underestimates the HSL solubilities.

Underestimation of aqueous solubilities of organic compounds by the UNIFAC method has been noted by Arbuckle, (1983) and Banerjee, (1985) for aromatic hydrocarbon and other groups of organic compounds in their determination of aqueous solubilities from activity coefficients. Banerjee, (1985) compared UNIFAC-derived aqueous solubilities with experimental values and derived an empirical equation based on the regression analysis of the data which is shown below.

$$
\log X^{\circ(h s I)}=1.20+0.782 \log \left(\frac{1}{\gamma_{i(a q) \text { UNIFAC }}^{\infty}}\right)
$$

The HSL solubility estimates using this corrected UNIFAC method are shown in Table 4. The HSL solubility estimates using the corrected UNIFAC method compare better than those of the UNIFAC method. The corrected UNIFAC method generally overestimates the values of the ratio method and is within a factor of three. Figure 7 graphically shows the comparison of the corrected UNIFAC method to the ratio method, and Figure 8 shows the improvement of the corrected UNIFAC method over the UNIFAC method. Although the corrected UNIFAC HSL estimate compares well with the ratio and melting point correction HSL estimates, it will not be used here. Empirical equations improving on the fit of the UNIFAC model to experimental data are abundant and specific to selected groups of compounds. But none of these corrected fit models 


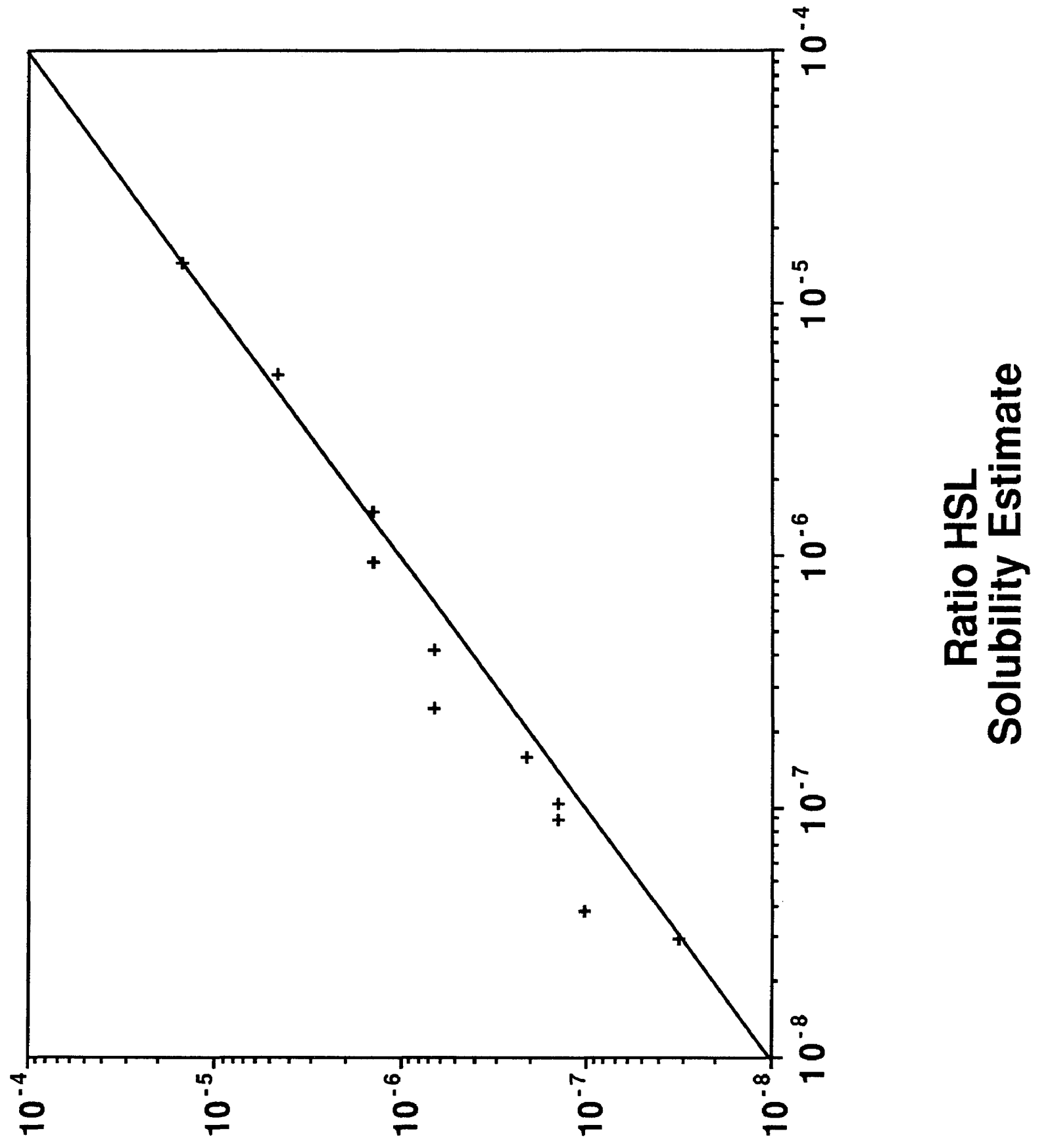

\section{әرеu!!s Kł!!!qnjos 7SH

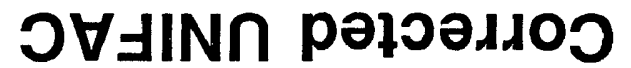




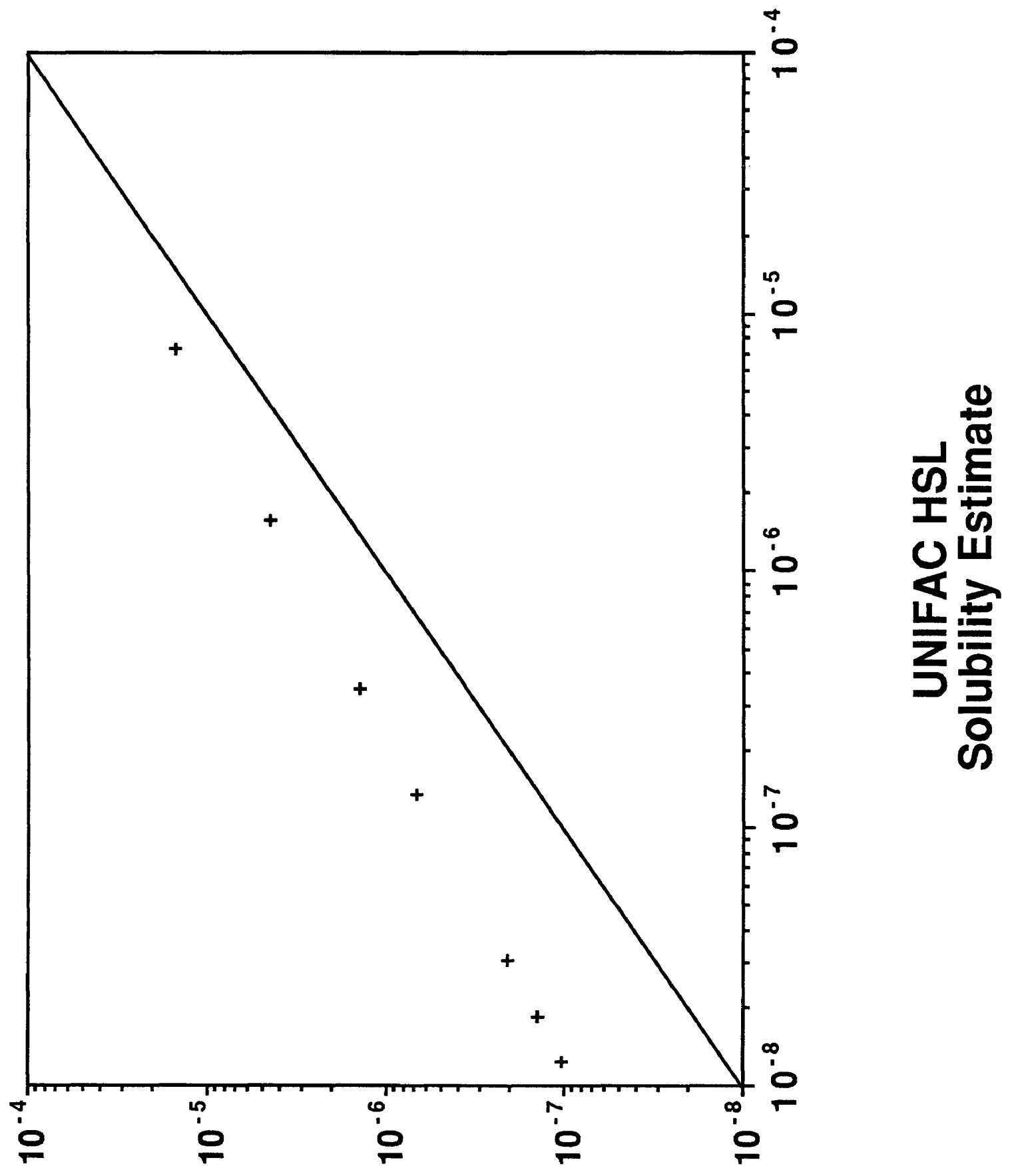

\section{әرeس!!s K!!!!qnjos 7SH

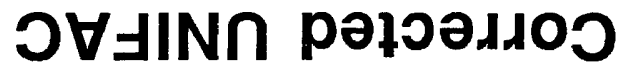


are as universally accepted or used as the UNIFAC model itself. Inclusion of other interaction parameters from the LLE or the VLE databases alone does not improve the correlation between UNIFAC-derived estimates of aqueous solubility and experimentally measured values for solid PAHs in water. Modified versions of UNIFAC using other parameters (Kikic et al, 1980 and Al-Sahhaf, 1989) show improved fit of the model to other groups of hydrocarbons. But these modifications to UNIFAC do not show an improved fit of the model (Gmehling et al, 1982) to aromatic hydrocarbons (Arbuckle, 1986). Improvements in the UNIFAC interaction parameters database used for calculating the activity coefficients of solid PAHs, or in the UNIFAC structural assumptions are needed, since severe underestimates have been found. For example, anthracene and phenanthrene have the same molecular weight and three aromatic ring structures, but quite different melting points and aqueous solubilities. UNIFAC-derived activity coefficients predicted for these two different compounds are the same. The UNIFAC model is unable to distinguish between them. The model works well in predicting the behavior of linear hydrocarbons; the correlation between the activity coefficient and the number of carbon atoms is very good (Fredenslund et al, 1975). Single ring aromatic compounds including alkyl substitutions and compounds with two ring structures are also predicted well, but as the number of 
aromatic rings increase beyond two the model shows large deviations in its ability to predict accurately, activity coefficients which are reliable. For example, the HSL solubility estimate of chrysene in Table 4, as predicted by the UNIFAC model, is an order of magnitude lower than the results of other methods of HSL estimation.

\section{Binary Mixture Experiments}

Experimental data for four binary liquid hydrocarbon mixtures (naphthalene/, anthracene/, pyrene/, and phenanthrene/benzene) equilibrated with water are shown in Tables 5a-d. Each table contains a series of concentrations for each $\mathrm{PAH}$ mixture which are shown graphically in Figures 9 - 12. "Ideal" solvent solubilities from Table 2 are included to indicate the maximum solute mole fraction in benzene, above which a solid phase would be formed. Pyrene and anthracene mixtures demonstrate this behavior as seen in Table $5 b$ and 5c. The phenanthrene mixtures had solids forming at the boundary between phases at mole fractions below the maximum solubility of phenanthrene in benzene. It is unknown in light of previous precautions why phenanthrene recrystallization occurred at $42-67$ percent below the maximum concentration for the solute in the hydrocarbon phase. The organic mixtures containing benzene and phenanthrene did not have solids present before injection into the equilibration vessels. Analysis of the mixture 
Table 5a. Solubility of naphthalene in aqueous and hydrocarbon phases from binary mixtures series at $25^{\circ} \mathrm{C}$

$\begin{array}{ccc}\begin{array}{c}\text { Aqueous Solubility } \\ (\mathrm{mg} / \mathrm{L})\end{array} & \begin{array}{c}\text { Hydrocarbon Phase } \\ \text { (mole fraction) }\end{array} & \begin{array}{r}\text { UNIFAC } \\ \boldsymbol{\gamma}_{\text {i(h) }}\end{array} \\ 5.29 & 0.090 & 0.960 \\ 9.93 & 0.129 & 0.964 \\ 17.96 & 0.198 & 0.970 \\ 26.24 & 0.261 & 0.975 \\ - & 0.294^{\mathrm{b}} & -\end{array}$

b - max solid solubility

Table 5b. Solubility of anthracene in water and in hydrocarbon phase from the series binary mixtures at $25^{\circ} \mathrm{C}$

Aqueous Solubility $(\mathrm{mg} / \mathrm{L})$

0.0052

0.0194

0.0283

0.0412

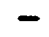

0.0325
Hydrocarbon Phase (mole fraction)

0.0040

0.0135

0.0197

0.0232

$0.024^{\mathrm{b}}$

$0.0251^{\mathrm{a}}$
UNIFAC

$\boldsymbol{\gamma}_{\mathrm{i}(\mathrm{hc})}$

0.841

0.845

0.847

0.849

0.849

- solids present in organic phase

b - max solid solubility 
Table 5c. Solubility of pyrene in aqueous and hydrocarbon phases from binary mixtures series at $25^{\circ} \mathrm{C}$

\begin{tabular}{|c|c|c|c|}
\hline $\begin{array}{c}\text { Aqueous } \\
\text { Solubility } \\
(m g / L)\end{array}$ & $\begin{array}{c}\text { Hydrocarbon } \\
\text { Phase } \\
\text { mole fraction } \\
\text { post } \\
\end{array}$ & $\begin{array}{c}\text { Hydrocarbon } \\
\text { Phase } \\
\text { mole fraction } \\
\text { prior } \\
\end{array}$ & $\begin{array}{l}\text { UNIFAC } \\
\gamma_{\mathrm{i}(\mathrm{hc})}\end{array}$ \\
\hline 0.021 & 0.029 & n.d. & 0.913 \\
\hline 0.038 & 0.051 & n.d. & 0.915 \\
\hline 0.039 & 0.051 & n.d. & 0.915 \\
\hline 0.061 & 0.074 & n.d. & 0.917 \\
\hline- & $0.093^{b}$ & - & - \\
\hline 0.074 & $0.093^{a}$ & 0.092 & 0.919 \\
\hline 0.084 & $0.123^{\mathrm{a}}$ & n.d. & 0.923 \\
\hline $\begin{array}{l}\text { - solids } p \\
\text { - max. sol } \\
\text {.d. - not }\end{array}$ & $\begin{array}{l}\text { in phase } \\
\text { ubility } \\
\text { ned }\end{array}$ & & \\
\hline
\end{tabular}

Table 5d. Solubility of phenanthrene in aqueous and hydrocarbon phases from binary mixture series at $25^{\circ} \mathrm{C}$

\begin{tabular}{c}
$\begin{array}{c}\text { Aqueous } \\
\text { Solubility } \\
(\mathrm{mg} / \mathrm{L})\end{array}$ \\
\hline 0.081 \\
0.255 \\
0.434 \\
0.500 \\
0.706 \\
-
\end{tabular}

\begin{tabular}{c}
$\begin{array}{c}\text { Hydrocarbon } \\
\text { Phase } \\
\text { mole fraction } \\
\text { post }\end{array}$ \\
\hline 0.023 \\
0.052 \\
$0.079^{\mathrm{a}}$ \\
$0.141^{\mathrm{a}}$ \\
$0.142^{\mathrm{a}}$ \\
$0.245^{\mathrm{b}}$
\end{tabular}

\begin{tabular}{c}
$\begin{array}{c}\text { Hydrocarbon } \\
\text { Phase } \\
\text { mole fraction } \\
\text { prior }\end{array}$ \\
\hline n.d. \\
0.060 \\
0.117 \\
0.156 \\
n.d. \\
-
\end{tabular}

UNIFAC

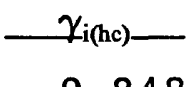

0.848

0.859

0.869

0.889

0.890

\footnotetext{
- solids present in the phase

b - max solid solubility

n.d. - not determined
} 
prior to injection and after equilibration did not show a large decrease in the phenanthrene mole fraction in the hydrocarbon phase to account for solid formation. A small drop in phenanthrene mole fraction occurred as expected, due to vaporization of benzene and to its high aqueous solubility, but this was not enough to cause the observed phenanthrene crystallization. The other binary mixtures did not demonstrate this behavior. UNIFAC-derived activity coefficients, $\gamma_{\mathrm{i}(\mathrm{hc})}$, calculated for each binary mixture increase with increasing solute mole fraction concentration. These $\gamma_{\mathrm{i}(\mathrm{hc})}$ approach unity as expected, but binary mixtures are not ideal.

In Figures 9 - 12, experimental aqueous solubility data for the four liquid hydrocarbon mixtures in Table 5a-d are compared to the solubility predictions from equation 9. Equation 9 uses the HSL solubility estimates from Table 4, the "ideal" solvent solubilities in Table 2, and assumes ideal solution behavior, $\gamma_{\mathrm{i}(\mathrm{hc})}=1$, to predict aqueous solubility. For a range of solute concentrations in binary mixtures, the experimental aqueous concentrations should fall on a line, which represents ideal behavior of the solute hydrocarbon and aqueous phase concentrations. This line is defined as the ratio HSL estimate (solid line). The melting point correction (heavy dash line) and the UNIFAC (dash - dot line) estimates are also shown. The corrected UNIFAC method of HSL solubility estimation is not 


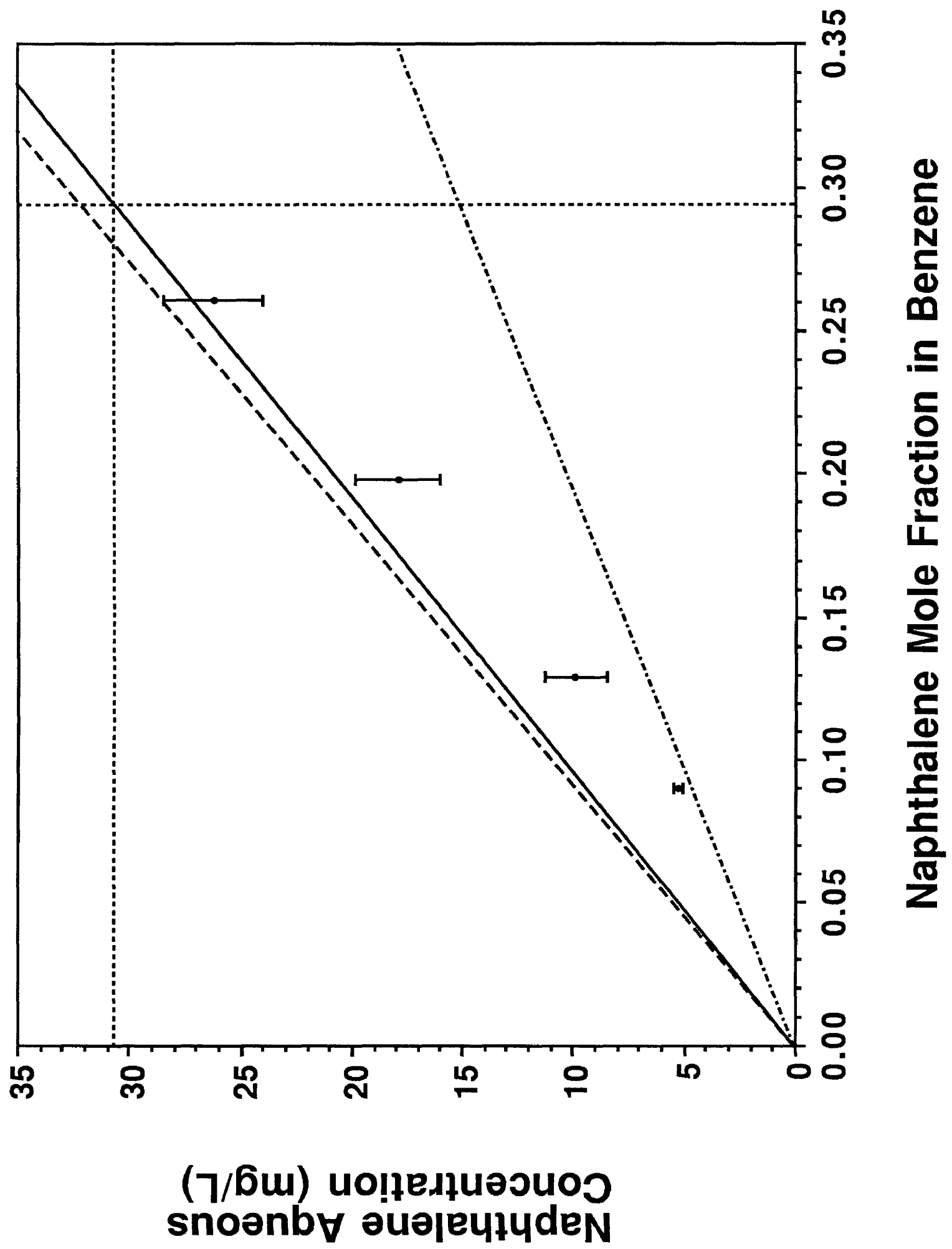




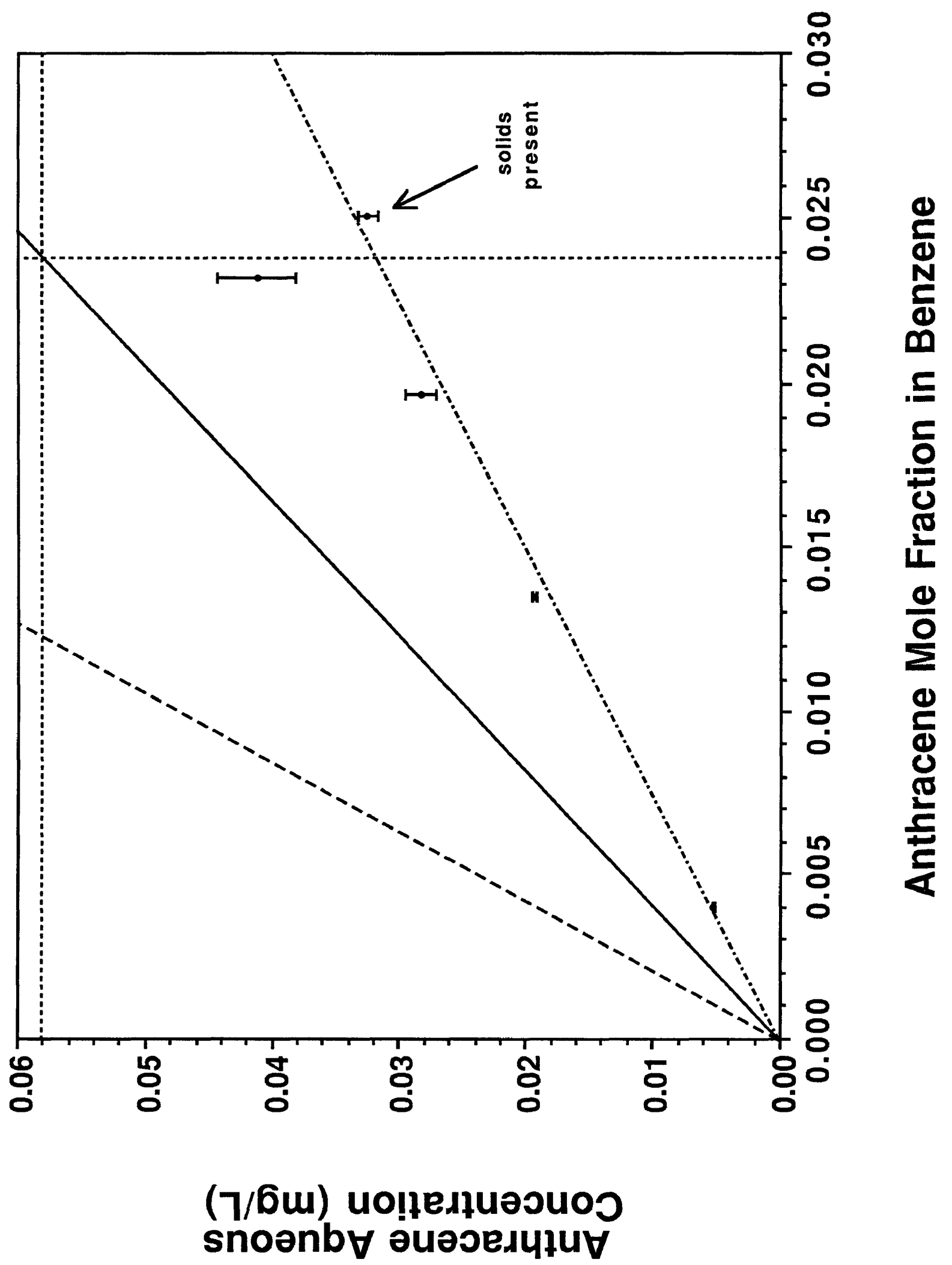




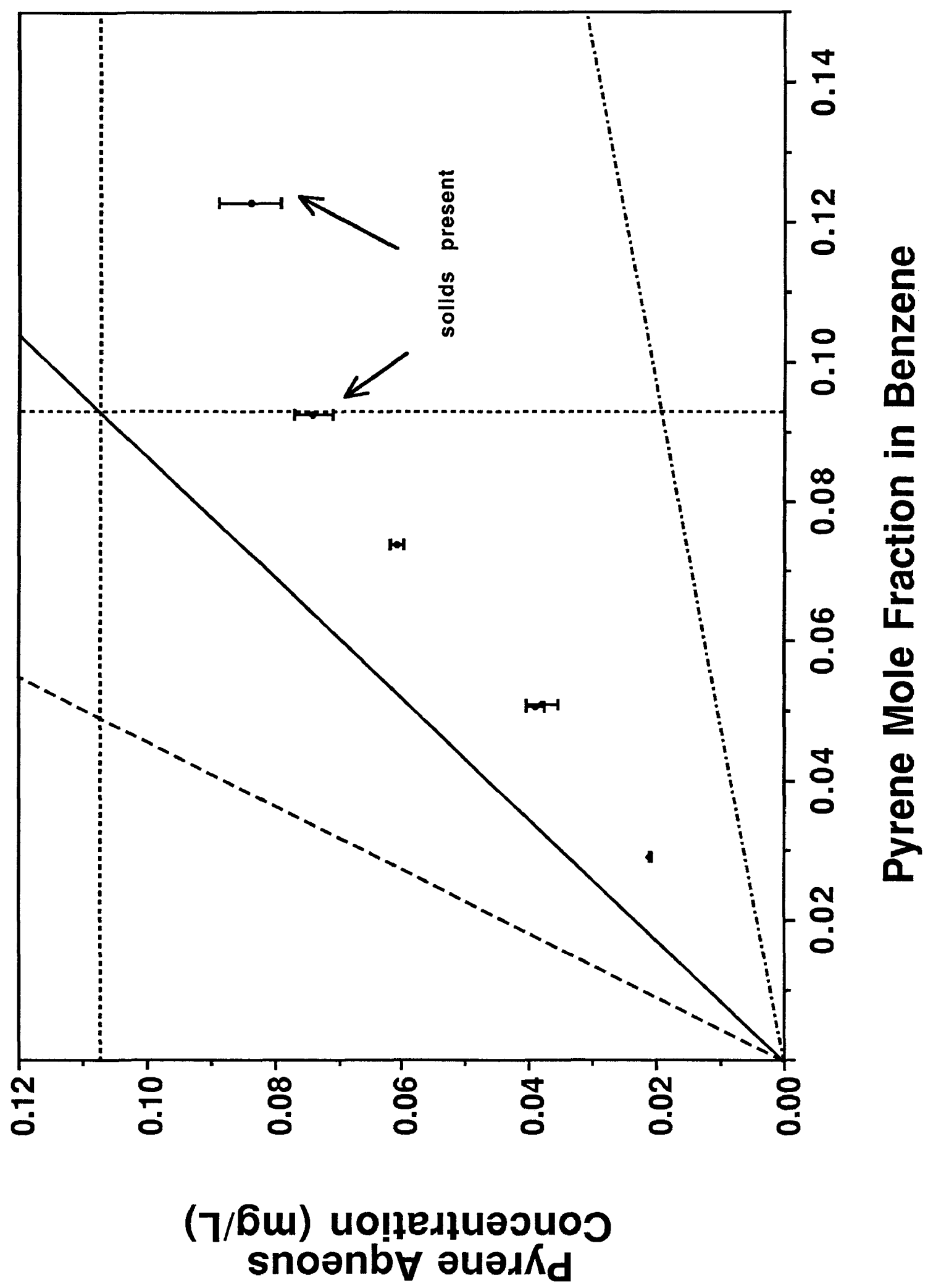




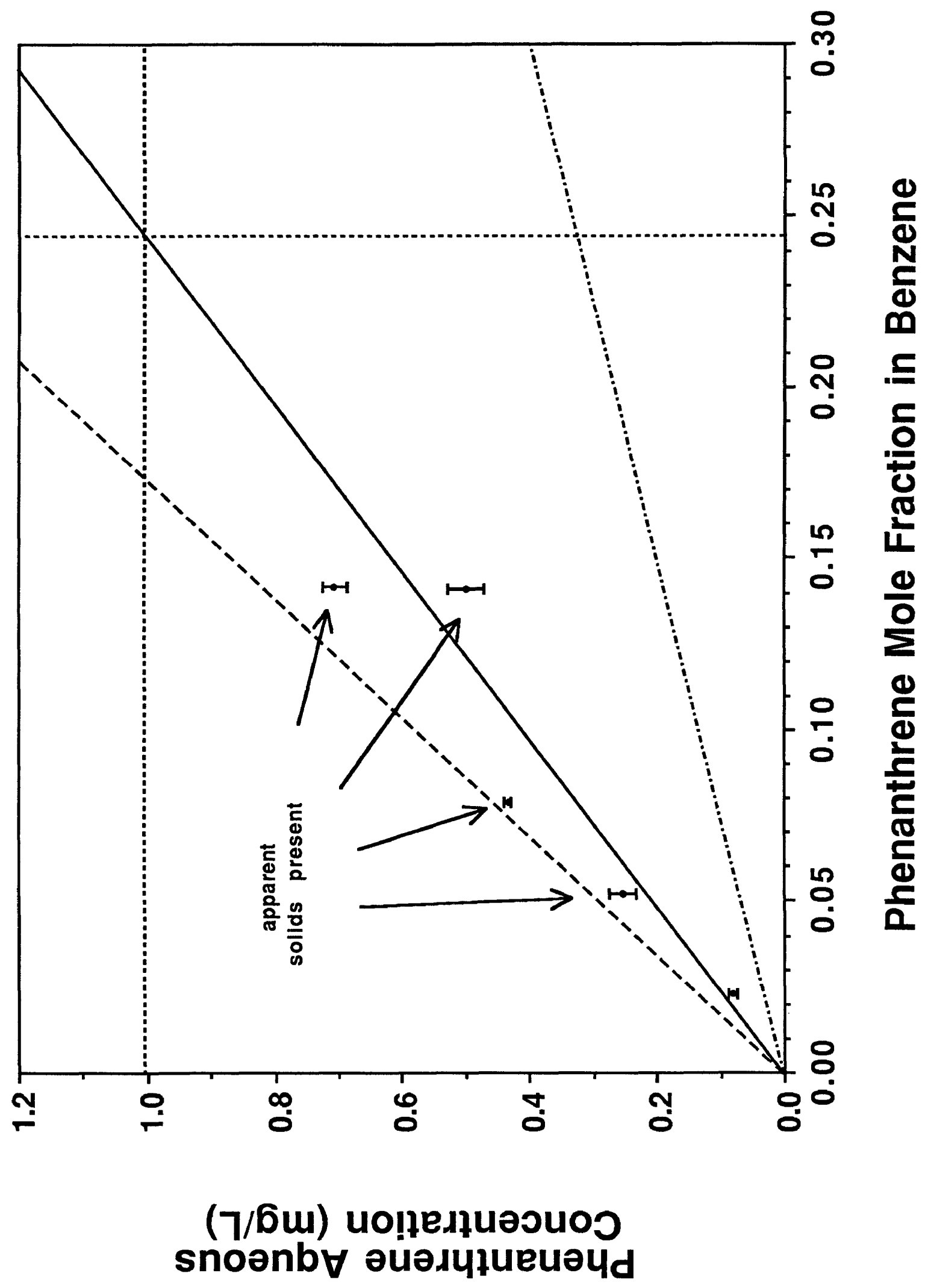


shown. The small dash lines are maximum compound solubilities in water and benzene.

The experimental data for each binary mixture falls below the predicted values from the ratio HSL solubility estimation method (solid line in Figures 9 - 11). This implies that the activity coefficient for the PAHs in benzene may be less than unity (i.e., the aromatic hydrocarbon mixtures may not be ideal as assumed in the ratio HSL method) - UNIFAC-derived $\gamma_{\mathrm{i}(\mathrm{hc})}$ values calculated for these aromatic hydrocarbon binary mixtures are less than unity, they range from 0.8 to near 1.0 (see Table 5). Aqueous solubilities predicted using activity coefficients that are ideal $\left(\gamma_{\mathrm{i}(\mathrm{hc})}=1\right)$ and calculated from UNIFAC $\left(\gamma_{\mathrm{i}(\mathrm{hc}) \text { UNIFAC }}\right)$ from Table 5 are shown in Table 6. The UNIFAC $\gamma_{\mathrm{i}(\mathrm{hc})}$ 's predict the aqueous phase concentrations for the hydrocarbon mixtures better than the $\gamma_{\mathrm{i}(\mathrm{hc})}$ 's assuming ideal behavior. Predicted aqueous phase concentration is still higher than experimentally obtained values. This coincides with the deviation of the experimental results from the ideal line. However, for simplicity the UNIFAC-derived $\gamma_{\mathrm{i}(\mathrm{hc})}$ values were not used in estimating the PAH aqueous phase concentrations for these mixtures.

The aqueous experimental results for the phenanthrene/benzene mixture in water, shown in Figure 12, are close to the predicted aqueous concentrations using the HSL solubility estimate derived from the ratio method. The 
年

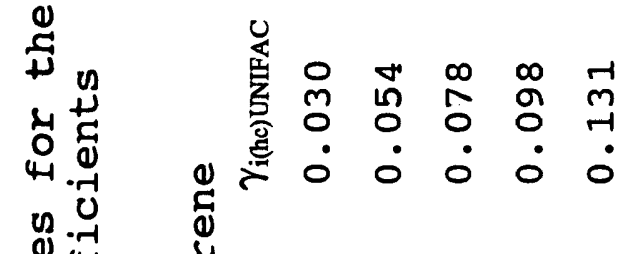

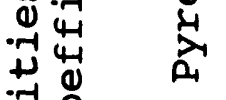

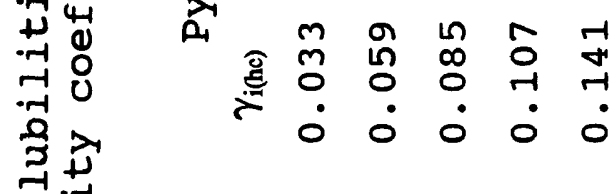

on

n

ว

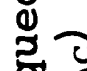

๒

\%

ช

$+$

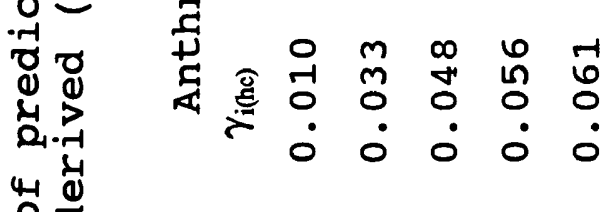

c

C

in

㟧

\%

兽

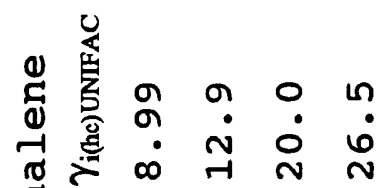

: $\tilde{\mathrm{A}}$

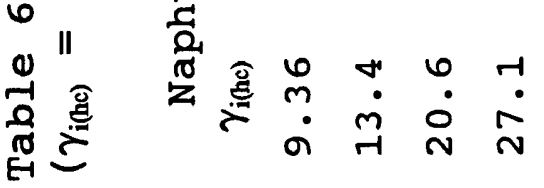


crystals of the PAH present in these mixtures may be responsible for the close fit of the experimental results to the predicted values. It is not understood why solids are present in the phenanthrene/benzene mixtures. The presence of a third phase (solids) in the experiment complicates the thermodynamics and is not accounted for in these estimates. The melting point correction estimate (heavy dash line) using Equation 22 gives an aqueous solubility that tends to overestimate the experimentally determined aqueous concentrations. This overestimation could prove to be a problem in situations where the $\mathrm{PAH}$ mole fraction in the hydrocarbon mixture was near the high end of its possible range. In this case, aqueous phase concentration estimates which are above the pure compound solubility would occur. This is not possible, so in that situation it would be best to replace the solubility estimate by the pure compound (as a solid) solubility value as the estimated aqueous phase concentration.

The UNIFAC (dot-dash) line from equation 24 , indicates a large underestimate of the experimental aqueous concentration. Although UNIFAC is easy to use, this severe underestimation makes it useful only as a first approximation. Empirical corrections to the UNIFAC model are continually being derived for various groups of compounds. These corrections improve the fit of the UNIFAC model to aqueous solubility, but none are used here because 
of their variable nature.

\section{Multicomponent Mixture Experiments}

After comparing the HSL solubility estimates to the results of binary liquid hydrocarbon mixtures equilibrated with water, the next step was to equilibrate a multicomponent liquid hydrocarbon mixture with water, and compare experimental results to those predicted using the three HSL solubility estimates. This experiment using a hydrocarbon mixture containing several solid PAHs will investigate the effect of multiple solutes in the hydrocarbon phase upon individual PAH aqueous concentrations, and the change expected in the activity coefficients in the organic phase. Experimental solubilities of a seven and eight component hydrocarbon mixture and water system are shown in Table 7 and 8 , respectively. The results are compared to predicted values from equation 9 using the HSL solubility estimates from Table 4, the experimental hydrocarbon phase mole fraction, and the UNIFAC-derived activity coefficients for the respective mixture. As expected from the binary mixture data, the UNIFAC $\gamma_{\mathrm{i}(\mathrm{hc})}$ are less than one. The results from Table 7 and 8 indicate that the ratio and melting point correction methods generally overestimate, and the UNIFAC method underestimates the aqueous phase concentrations of the PAHs. This is consistent with the binary mixture 


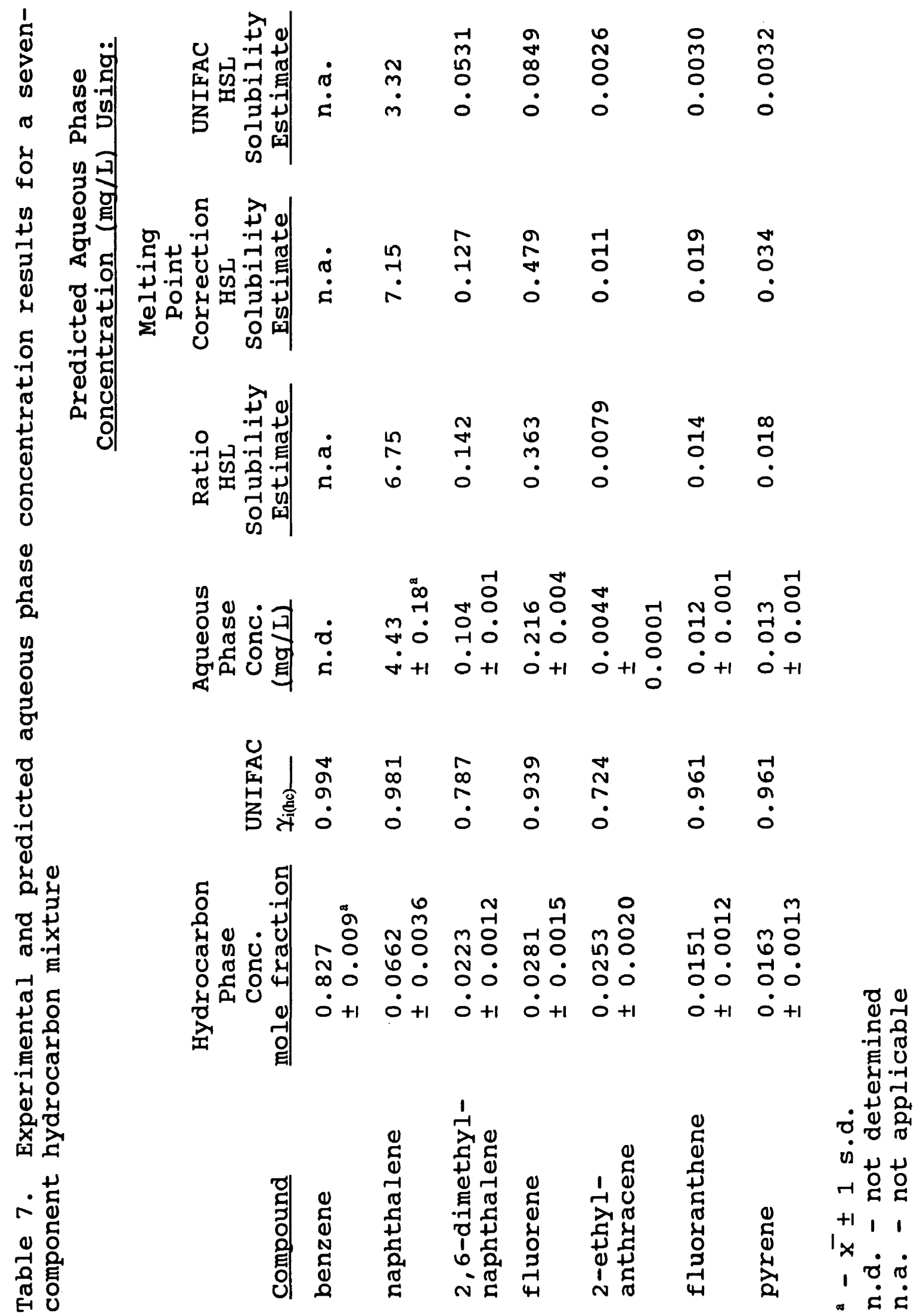




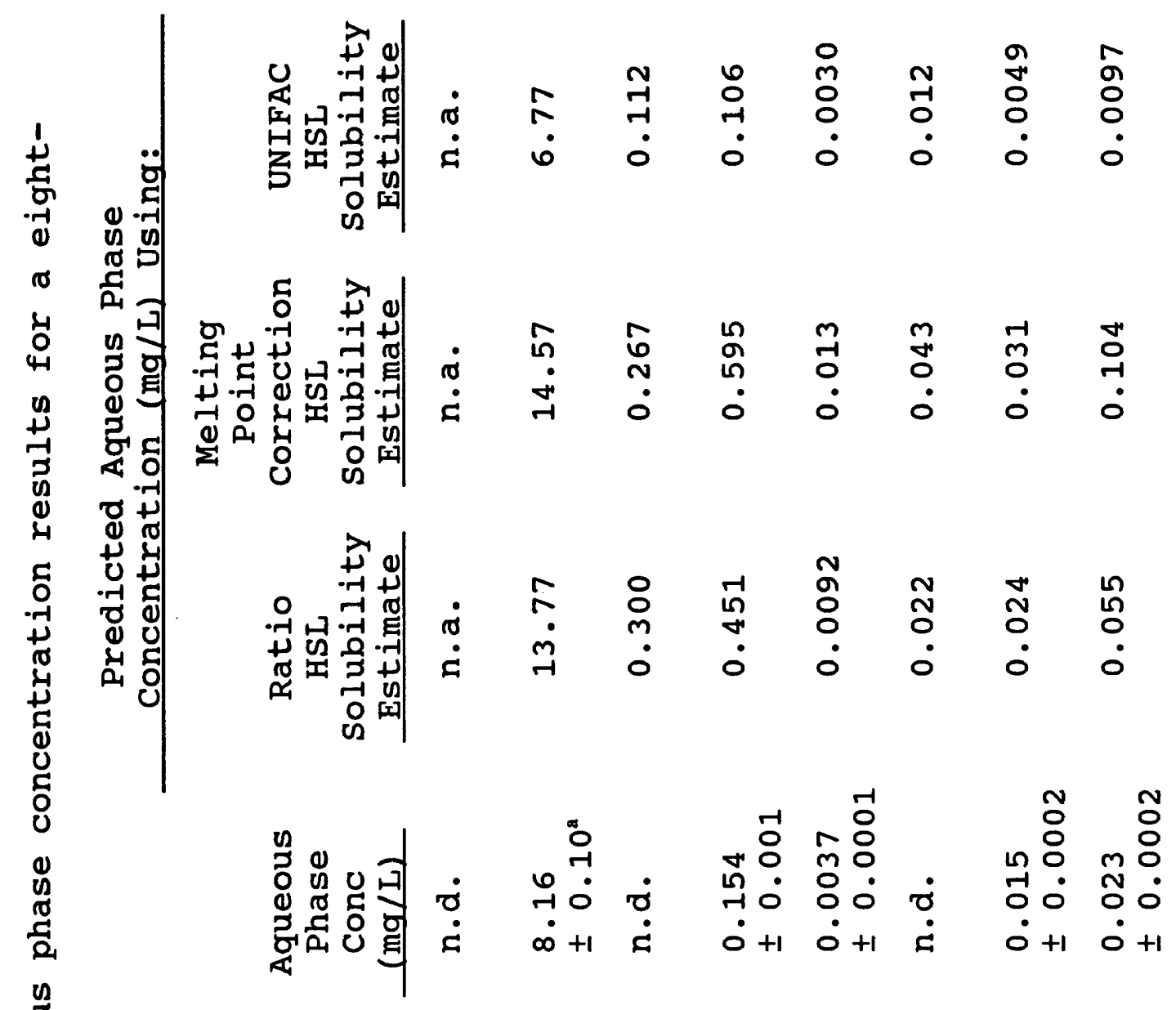

61

号

总

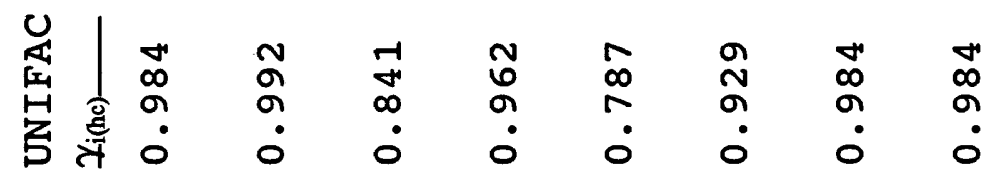

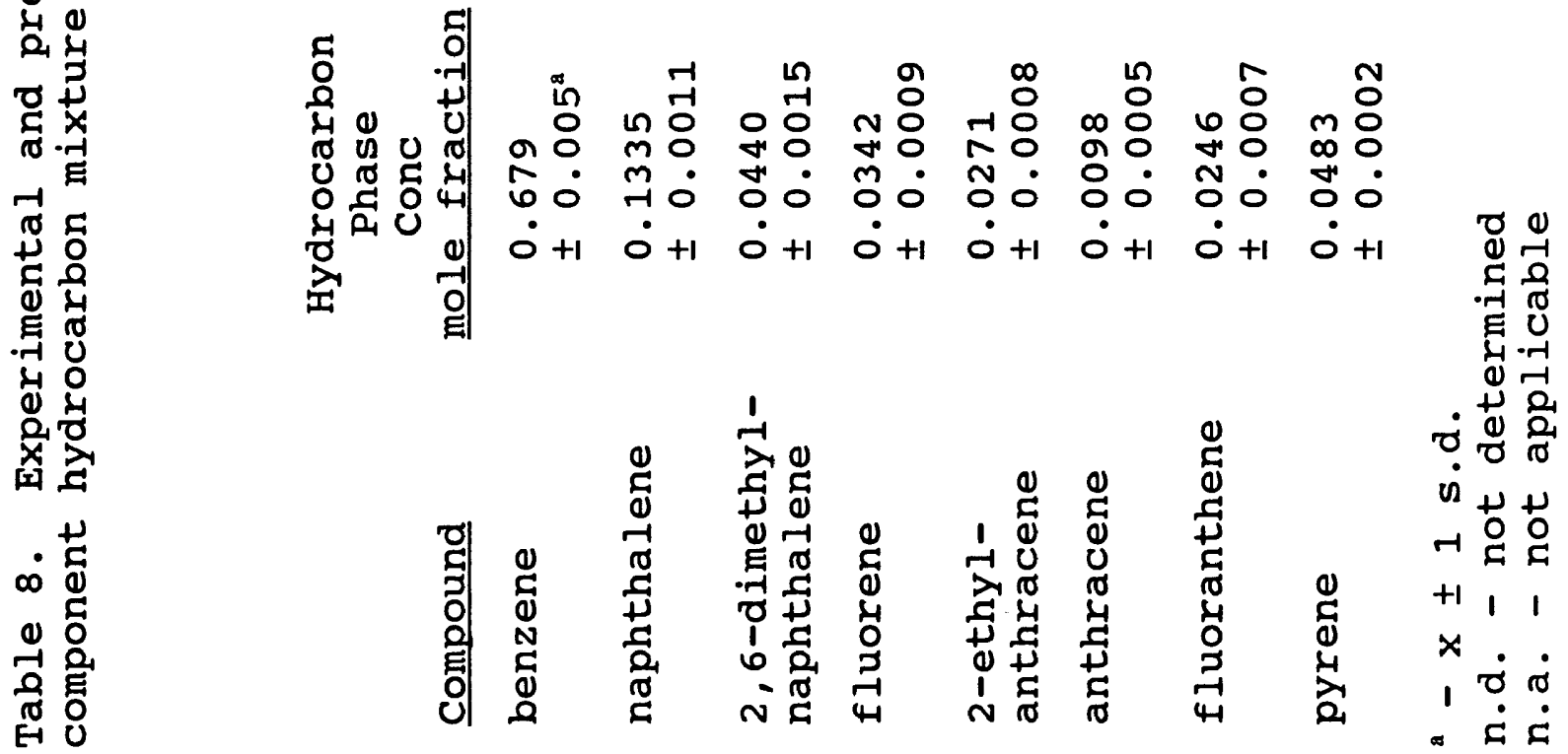


results.

The ratio and melting point correction methods of estimating HSL solubility overestimate the aqueous phase concentrations in binary and multicomponent mixtures. The ratio method appears to be accurate within a factor of three and the melting point correction method appears within a factor of four according to the experimental mixture results. The UNIFAC method of estimating HSL solubility yields underestimates of the aqueous phase concentration by a factor of four or more in some cases. From a practical perspective, the ratio method of estimating HSL solubility will not be routinely used for environmental work since additional experimental data are required. The melting point correction method generally does not require additional experimental work since pure compound solubilities of many solid hydrocarbons of environmental concerns are available. In contrast, use of the UNIFAC method only requires a computer, appropriate software, and knowledge of the system component chemical structures.

\section{Solid Solubility Kinetics}

The appearance of solid solute in undersaturated organic mixtures in the equilibration experiments was a curious phenomenon that required further investigation.

Phenanthrene/benzene and pyrene/benzene hydrocarbon mixtures near their maximum solute solubility were each equilibrated 
with water at concentrations where solids were induced at the organic - aqueous phase boundary. once the solids had formed, usually overnight, the reversal of the process of solute crystallization was attempted by mechanical means. This was done with the consideration that the observed crystals might not be at true thermodynamic equilibrium because of slow kinetics. The organic phase was analyzed prior to and after this experiment to assure that the benzene in the vapor phase had not escaped the bottle. Results are shown in Table 9. Simultaneous observations of shaken/unshaken and stirred/shaken sealed bottles of binary hydrocarbon mixtures in contact with water for 48 hours did not show any reversal of crystallization due to kinetic effects. Shaking the mixture bottles did force the crystal layer from its normal position in the bottom of the organic phase to the surface of the aqueous phase. Visual observation of crystal volume did not permit an accurate assessment of the mass of crystals present. Unfortunately, no practical means of isolation of the crystals for mass determination was apparent. Heating the bottles from room temperature $\left(25^{\circ} \mathrm{C}\right)$ to $45^{\circ} \mathrm{C}$ in an incubator overnight redissolved the crystals. However, after cooling to room temperature the solids recrystallized in their respective phase. It appears that the kinetics of the solute transport between phases does not cause recrystallization, but a surface effect at the boundary of the aqueous and organic 
Table 9. Solubility of selected PAHs in hydrocarbon phases equilibrated with water containing solids

\begin{tabular}{|c|c|c|c|}
\hline Compound & Action & $\begin{array}{c}\text { Solubility } \\
\text { mole fraction } \\
\text { prior }\end{array}$ & $\begin{array}{l}\text { Solubility } \\
\text { mole fraction } \\
\text { post }\end{array}$ \\
\hline Phenanthrene & not shaken & 0.160 & 0.147 \\
\hline Phenanthrene & shaken & 0.150 & 0.138 \\
\hline Pyrene & not shaken & 0.064 & 0.049 \\
\hline Pyrene & shaken & 0.066 & 0.047 \\
\hline Phenanthrene & stirred & 0.191 & 0.144 \\
\hline Phenanthrene & shaken & 0.190 & 0.153 \\
\hline
\end{tabular}


phases may contribute to this phenomenon. Investigation of surface processes is outside the scope of this study, but the author suggests that crystal formation, quantity, composition and thermodynamic (phase) properties should be investigated in further research.

\section{Creosote Experiment}

The HSL solubility estimation procedures have been evaluated using simple hydrocarbon mixtures equilibrated with water. An attempt to compare an actual hydrocarbon mixture results with these estimates was done. creosote contains many solid components in very low mole fraction concentrations, and when equilibrated with water, produce very low aqueous concentrations. Separation and identification of the components in the aqueous phase are difficult. Methodology requires large volume extraction, concentration, and GC analysis to accurately determine the aqueous concentrations. The HPLC techniques used during this study for analysis were untested when dealing with a complex mixture like creosote.

Pure creosote was diluted with benzene and equilibrated with water at $25^{\circ} \mathrm{C}$ for 24 hours and an attempt made to analyze the components. Analysis of hydrocarbon and aqueous phases by methods used here did not produce results that could be compared to HSL methods of estimation. Determining mole fractions of individual components in a complex mixture 
such as creosote with over 250 components is a formidable task even with the aid of a mass spectrometer. And HPLC analysis of the water soluble components proved to be an inappropriate method of analysis. For analyzing a complex mixture such as creosote, different techniques must be employed that can separate individual compounds for identification, and concentrate components for quantitation using extraction techniques.

\section{Summary}

For many environmental exposure estimates, accuracy of the predicted aqueous phase concentrations of hydrocarbon within a factor of two is sufficient. It is also prudent, in most cases, to be conservative in estimates of exposure. The melting point correction method for estimating HSL solubility may be the method of choice (i.e., provides an overestimate) since it is generally conservative in estimating exposure and is reasonably accurate within a factor of four. In addition, the melting point correction method (equation 22) is thermodynamically derived, and therefore, the coefficient of 0.01 and the system temperature $\left(25^{\circ}\right)$ can be adjusted for systems at different temperatures without further experimentation. However, care must be taken to ensure that the estimate does not exceed the water solubility of the pure compound. 


\section{CONCLUSIONS}

The hypothetical supercooled liquid solubility is required for estimating the aqueous phase concentration of hydrocarbons which are solids in their pure state at the system temperature when they are components of a liquid hydrocarbon mixtures. Three methods of estimating HSL solubility have been evaluated using experimental aqueous solubilities of liquid hydrocarbon mixtures containing polynuclear aromatic hydrocarbons. A simple equation requiring only the melting point and pure compound aqueous solubility of the solid is the method of choice since: 1) additional experiment work would generally not be required; 2) the estimate would most likely be conservative; and 3) the estimate would most likely be accurate within a factor of 4 .

The presence of a solid phase in an undersaturated solution at equilibrium leaves a question unanswered in this study. The reasons for this unstable system to persist is unknown. Surface chemistry effects at the molecular level or phase boundary conditions might be the cause of this phenomenon as the solute moves from aqueous to organic phase. This effect only seems to apply to phenanthrene.

Identifying the behavior of the solids in an actual hydrocarbon mixture found in the environment is another 
possible extension to explore. The methodology used here is limited for such applications, but the use of these estimates may be used in certain field studies. 


\section{LITERATURE CITED}

Al-Sahhaf, T.A. 1989. Prediction of the solubility of Hydrocarbons in Water using UNIFAC. J. Environ. Sci. Health. A24(1), 49-56.

American Petroleum Institute. 1981. Selected Values of Properties of Hydrocarbons and Related Compounds, Table 23-2, API Research Project 44, Supplementary Vol. No. A-81. Thermodynamic Research Center, Texas A \& M, College station, TX.

Arbuckle, W.B. 1983. Estimating Activity Coefficient for Use in Calculating Environmental Parameters. Environ. Sci. Technol. 17(9), 537-542.

Arbuckle, w.B. 1986. Using UNIFAC to Calculate Aqueous Solubilities. Environ. Sci. Technol. 20(10), 1060 -1064 .

Banerjee, S. 1984. Solubility of Organic Mixtures in Water. Environ. Sci. Technol. 18(8), 587-591.

Banerjee, S. 1985. Calculation of the water solubility of Organic Compounds with UNIFAC - Derived Parameters. Environ. Sci. Technol. 19(4), 369-370.

Banerjee, S., S.H. Yalkowsky, and S.C. Valvani. 1980. Water Solubility and octanol - Water Partition Coefficient of Organics. Limitations of Solubility Partition Coefficient Correlation. Environ. Sci. Technol. 14(10), 1227-1229.

Billington, J.W., G.L. Huang, F. Szeto, W.Y. Shiu, and D. Mackay. 1988. Preparation of Aqueous Solutions of Sparingly Soluble Organic Substances:

II. Multicomponent Systems - Hydrocarbon Mixtures and Petroleum Products. Environ. Toxicol. Chem. 7, 125 -137 .

Burris, D.R. and W.G. MacIntyre. 1985. Water Solubility Behavior of Binary Hydrocarbon Mixtures. Environ. Toxicol. Chem. 4, 371-377. 
Burris, D.R. and W.G. MacIntyre. 1986. Thermodynamic Study of Solutions of Liquid Hydrocarbon Mixtures in Water. Geochimica et Cosmochimica Acta, 50, 1545-1549.

Burris, D.R. and W.G. MacIntyre. 1987. Water Solubility Behavior of Hydrocarbon Mixtures - Implications for Petroleum Dissolution in oil in Freshwater: Chemistry, Biology, Countermeasure Technology. Vandermeulen, J.H. and S.E. Hrudey, eds. Pergamon Press, New York.

Chiou, C.T., V.H. Freed, D.W. Schmedding, and R.L. Kohnert. 1977. Partition Coefficient and Bioaccumulation of Selected Organic Chemicals. Environ. Sci. Technol. $11(5), 475-478$.

DeVoe, H., M.M. Miller, and S.P. Wasik. 1980. Generator Columns and High Pressure Liquid Chromatography for Determining Aqueous Solubilities and Octanol - water Partition Coefficients of Hydrophobic Substances. Jour of Research of the NBS. 86(4), 361-366.

Dickhut, R.M., A.W. Andren, and D.E. Armstrong. 1986. Aqueous Solubilities of Six Polychlorinated Biphenyl Congeners at Four Temperatures. Environ. Sci. Technol. $20(8), 807-810$.

Eganhouse, R.P. and J.A. Calder. 1976. The Solubility of Medium Molecular Weight Aromatic Hydrocarbons and the Effects of Hydrocarbon Co-solutes and Salinity. Geochimica et Cosmochimica Acta. 40, 555-561.

Fredenslund, A., R.L. Jones, and J.M. Prausnitz. 1975. Group-Contribution Estimation of Activity Coefficients in Nonideal Liquid Mixtures. AIChE Jour. 21, 1086 -1099 .

Gmehling, J., P. Rasmussen, and A. Fredenslund. 1982. Vapor-Liquid Equilibria by UNIFAC Group Contribution. Revision and Extension. 2. Ind. Eng. Ch. Process Des. Dev. 21(1), 118-127.

Grob, R.S. 1977. Modern Practice of Gas Chromatography. John Wiley \& Sons, New York, NY.

Hildebrand, J.H. and R.L. Scott. 1962. Reqular solutions. Prentice - Hall, Inc., Englewood, N.J. 
Kenaga, E.E. and C.A.I. Goring. 1980. Relationship Between Water Solubility, Soil sorption, Octanol-Water

Partitioning, and Concentration of Chemicals in Biota. Eaton, J.G., P.R. Parrish, and A.C. Hendricks, eds. Aquatic Toxicology, ASTM STP 707, American Society for Testing and Materials, 78-115.

Kikic, I., P. Alessi, P. Rasmussen, and A. Fredenslund. 1980. On the Combinatorial Part of the UNIFAC and UNIQUAC Models. Can. Jour. Chem. Eng. 58, 253-258.

Leinonen, P.J. 1976. The Fate of Spilled Oil. PhD Thesis. University of Toronto, Canada.

Leinonen, P.J. and D. Mackay. 1973. The Multicomponent Solubility of Hydrocarbons in Water. Can. Jour. Chem. Eng. 51, 230-233.

Lu, Mu Zhen. 1982. Organic Compound Levels in a sediment Core from the Elizabeth River of Virginia. MA Thesis, Virginia Institute of Marine Science, Gloucester Point, VA.

Mackay, D. and W.Y. Shiu. 1977. Aqueous Solubility of Polynuclear Aromatic Hydrocarbons. Jour. Chem. Eng. Data. $22(4), 399-402$.

MaY, W.E., S.P. Wasik, and D.H. Freeman. 1978a. Determination of the Aqueous Solubility of Polynuclear Aromatic Hydrocarbons by a Coupled Column Liquid Chromatographic Technique. Anal. Chem. 50(1), 175-179.

MaY, W.E., S.P. Wasik, and D.H. Freeman. 1978b. Determination of the Solubility Behavior of some Polycyclic Aromatic Hydrocarbons in Water. Anal. Chem. $50(7), 997-1000$.

McLaughlin, E. and H.A. Zainal. 1959. The Solubility Behavior of Aromatic Hydrocarbons in Benzene. J. Chem. Soc. $177,863-867$.

Miller, M.M., S.P. Wasik, G.L. Huang, W.Y. Shiu, and D. Mackay. 1985. Relationship between Octanol - Water Partition Coefficient and Aqueous Solubility. Environ. Sci. Technol. 19(6), 522-529.

Neely, W.B., D.R. Branson, and G.E. Blau. 1974. Partition coefficient to Measure Bioconcentration Potential of Organic Chemicals in Fish. Environ. Sci. Technol. 8 (13), 1113-1114. 
Schwarz, F.P. 1977. Determination of Temperature Dependence of Solubilities of Polycyclic Aromatic Hydrocarbons in Aqueous Solutions by a Fluorescence Method. Jour. Chem. Eng. Data. $22(3)$, 273-277.

Schwarz, F.P. and S.P. Wasik. 1976. Fluorescence Measurements of Benzene, Naphthalene, Anthracene, Pyrene, Fluoranthene, and Benzo(e)pyrene in Water. Anal. Chem. 48(3), 524-528.

Shiu, W.Y., A. Maijanen, A.L.Y. Ng, and D. MacKay. 1988. Preparation of Aqueous Solutions of Sparingly Soluble Organic Substances: II. Multicomponent systems Hydrocarbon Mixtures and Petroleum Products. Environ. Toxicol. and Chem. 7, 125-137.

Tewari, Y.B., M.M. Miller, S.P. Wasik, and D.E. Mantire. 1982. Aqueous Solubility and Octanol/Water Partition Coefficient of Organic Compounds at.25.0 $0^{\circ} \mathrm{C}$ J. Chem. Eng. Data. 27, 451-454.

Velapoldi, R.A., P.A. White, W.E. May, and K.R. Eberhardt. 1983. Spectrofluorimetric Determination of Polycyclic Aromatic Hydrocarbons in Aqueous Effluents from Generator Columns. Anal Chem 55, 1896-1901.

Wauchope, R.D. and F.W. Getzen. 1972. Temperature Dependence of Solubilities in water and Heats of Fusion of Solid Aromatic Hydrocarbons. J. Chem. Eng. Data. $17,38-41$.

Weast, R.C., M.J. Astle, and W.H. Beyer, eds. 1984. CRC Handbook of Chemistry and Physics, 65th ed. CRC Press, Boca Raton, FL.

Whitehouse, B.G. 1984. The Effects of Temperature and Salinity on the Aqueous Solubility of Polynuclear Aromatic Hydrocarbons. Marine Chemistry, Elsevier Science Publishers B.V.

Yalkowsky, S.H. 1979. Estimation of Entropies of Fusion of Organic Compounds. Ind. Eng. Chem. Fund. 18(2), 108 -111 .

Yalkowsky, S.H. and S.C. Valvani. 1980. Solubility and Partitioning I: Solubility of Nonelectrolytes in water. Jour. Pharm. Sci. 69(8), 912-922.

Yalkowsky, S.H., S.C. Valvani, and D. Mackay. 1983. Estimation of the Aqueous Solubility of Some Aromatic Compounds. Residue Reviews. 85, 43-55. 
VITA

George G. Vadas

Born in Latrobe, Pennsylvania, January 10, 1956. Graduated from Derry Area Senior High School in 1974 . Earned a B.S. in Biology from The Pennsylvania state University in 1978. Entered the masters program in the College of William and Mary, school of Marine Science in 1988. Presently employed at the College of William and Mary, School of Marine science. 